Old Dominion University ODU Digital Commons

2014

\title{
Measurement of the Structure Function of the Nearly Free Neutron Using Spectator Tagging in Inelastic ${ }^{2} \mathrm{H}\left(\mathrm{e}, \mathrm{e}^{\prime} \mathrm{p}^{\Xi}\right)$ X Scattering with CLAS
}

S. Tkachenko

Old Dominion University

S.E. Kuhn

Old Dominion University, skuhn@odu.edu

J.Zhang

Old Dominion University

K.P. Adhikari

Old Dominion University

M.J. Amaryan

Old Dominion University

See next page for additional authors

Follow this and additional works at: https://digitalcommons.odu.edu/physics_fac_pubs

Part of the Elementary Particles and Fields and String Theory Commons, Plasma and Beam Physics Commons, and the Quantum Physics Commons

\section{Repository Citation}

Tkachenko, S.; Kuhn, S. E.; Zhang, J.; Adhikari, K. P.; Amaryan, M. J.; Klein, A.; Koirala, S.; Mayer, M.; Zana, L.; and CLAS

Collaboration, "Measurement of the Structure Function of the Nearly Free Neutron Using Spectator Tagging in Inelastic ${ }^{2} \mathrm{H}\left(\mathrm{e}, \mathrm{e}^{\mathrm{e}} \mathrm{p}^{\Sigma}\right) \mathrm{X}$

Scattering with CLAS" (2014). Physics Faculty Publications. 361.

https://digitalcommons.odu.edu/physics_fac_pubs/361

\section{Original Publication Citation}

Collaboration, C., Tkachenko, S., Baillie, N., Kuhn, S. E., Zhang, J., Arrington, J., . . Zonta, I. (2014). Measurement of the structure function of the nearly free neutron using spectator tagging in inelastic ${ }^{2} \mathrm{H}\left(\mathrm{e}, \mathrm{e}^{\prime} \mathrm{p}_{\mathrm{s}}\right) \mathrm{X}$ scattering with CLAS. Physical Review C, 89(4), 045206. doi:10.1103/PhysRevC.89.045206 
Authors

S. Tkachenko, S. E. Kuhn, J. Zhang, K. P. Adhikari, M. J. Amaryan, A. Klein, S. Koirala, M. Mayer, L. Zana, and CLAS Collaboration 


\section{Measurement of the structure function of the nearly free neutron using spectator tagging in inelastic} ${ }^{2} \mathrm{H}\left(e, e^{\prime} p_{s}\right) X$ scattering with CLAS

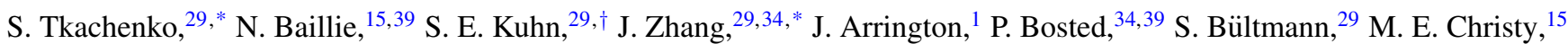
D. Dutta, ${ }^{41}$ R. Ent, ${ }^{34}$ H. Fenker, ${ }^{34}$ K. A. Griffioen, ${ }^{39}$ M. Ispiryan, ${ }^{42}$ N. Kalantarians, ${ }^{29,38}$ C. E. Keppel, ${ }^{15,34}$ W. Melnitchouk, ${ }^{34}$ V. Tvaskis, ${ }^{34}$ K. P. Adhikari, ${ }^{29}$ M. Aghasyan, ${ }^{18}$ M. J. Amaryan, ${ }^{29}$ S. Anefalos Pereira, ${ }^{18}$ H. Avakian, ${ }^{34}$ J. Ball, ${ }^{7}$

N. A. Baltzell, ${ }^{1,33}$ M. Battaglieri, ${ }^{19}$ I. Bedlinskiy, ${ }^{22}$ A. S. Biselli, ${ }^{5,11}$ W. J. Briscoe, ${ }^{14}$ W. K. Brooks, ${ }^{34,37}$ V. D. Burkert, ${ }^{34}$ D. S. Carman, ${ }^{34}$ A. Celentano, ${ }^{19}$ S. Chandavar, ${ }^{28}$ G. Charles, ${ }^{7}$, P. L. Cole,${ }^{16}$ M. Contalbrigo, ${ }^{17}$ O. Cortes, ${ }^{16}$ V. Crede, ${ }^{13}$ A. D’Angelo, ${ }^{20,31}$ N. Dashyan, ${ }^{40}$ R. De Vita, ${ }^{19}$ E. De Sanctis, ${ }^{18}$ A. Deur, ${ }^{34}$ C. Djalali, ${ }^{33}$ G. E. Dodge, ${ }^{29}$ D. Doughty, ${ }^{8,34}$ R. Dupre ${ }^{21}$ H. Egiyan, ${ }^{26,34}$ A. El Alaoui, ${ }^{1, \S}$ L. El Fassi, ${ }^{1, \|}$ L. Elouadrhiri, ${ }^{34}$ P. Eugenio, ${ }^{13}$ G. Fedotov, ${ }^{32,33}$ J. A. Fleming, ${ }^{10}$ B. Garillon, ${ }^{21}$ N. Gevorgyan, ${ }^{40}$ Y. Ghandilyan, ${ }^{40}$ G. P. Gilfoyle, ${ }^{36}$ K. L. Giovanetti, ${ }^{23}$ F. X. Girod, ${ }^{7,34}$ J. T. Goetz, ${ }^{28}$ E. Golovatch, ${ }^{32}$ R. W. Gothe, ${ }^{33}$ M. Guidal, ${ }^{21}$ L. Guo, ${ }^{12}$ K. Hafidi, ${ }^{1}$ H. Hakobyan, ${ }^{37,40}$ C. Hanretty, ${ }^{38,}, \mathrm{I}$ N. Harrison, ${ }^{9}$ M. Hattawy, ${ }^{21}$ K. Hicks, ${ }^{28}$ D. Ho, ${ }^{5}$ M. Holtrop,${ }^{26}$ C. E. Hyde, ${ }^{29}$ Y. Ilieva, ${ }^{14,33}$ D. G. Ireland,${ }^{35}$ B. S. Ishkhanov, ${ }^{32}$ H. S. Jo, ${ }^{21}$ D. Keller, ${ }^{38}$ M. Khandaker, ${ }^{27}$ A. Kim, ${ }^{24}$ W. Kim, ${ }^{24}$ P. M. King, ${ }^{28}$ A. Klein, ${ }^{29}$ F. J. Klein, ${ }^{6}$ S. Koirala, ${ }^{29}$ V. Kubarovsky, ${ }^{30,34}$ S. V. Kuleshov, ${ }^{22,37}$ P. Lenisa, ${ }^{17}$ S. Lewis, ${ }^{35}$ K. Livingston, ${ }^{35}$ H. Lu,,${ }^{5,33}$ M. MacCormick, ${ }^{21}$ I. J. D. MacGregor, ${ }^{35}$ N. Markov, ${ }^{9}$ M. Mayer, ${ }^{29}$ B. McKinnon, ${ }^{35}$ T. Mineeva, ${ }^{9}$ M. Mirazita, ${ }^{18}$ V. Mokeev, ${ }^{32,34}$ R. A. Montgomery, ${ }^{35}$ H. Moutarde, ${ }^{7}$

C. Munoz Camacho, ${ }^{21}$ P. Nadel-Turonski, ${ }^{14,34}$ S. Niccolai, ${ }^{21}$ G. Niculescu, ${ }^{23}$ I. Niculescu, ${ }^{23}$ M. Osipenko, ${ }^{19}$ L. L. Pappalardo, ${ }^{17}$ R. Paremuzyan, ${ }^{40, \ddagger}$ K. Park, ${ }^{24,34}$ E. Pasyuk, $, 2,34$ J. J. Phillips, ${ }^{35}$ S. Pisano, ${ }^{18}$ O. Pogorelko, ${ }^{22}$ S. Pozdniakov, ${ }^{22}$ J. W. Price, ${ }^{3}$

S. Procureur, ${ }^{7}$ D. Protopopescu, ${ }^{35}$ A. J. R. Puckett, ${ }^{9}$ D. Rimal, ${ }^{12}$ M. Ripani, ${ }^{19}$ A. Rizzo, ${ }^{20, \#}$ G. Rosner, ${ }^{35}$ P. Rossi, ${ }^{18,34}$ P. Roy, ${ }^{13}$ F. Sabatié, ${ }^{7}$ D. Schott, ${ }^{14}$ R. A. Schumacher, ${ }^{5}$ E. Seder, ${ }^{9}$ I. Senderovich, ${ }^{2}$ Y. G. Sharabian, ${ }^{34}$ A. Simonyan, ${ }^{40}$ G. D. Smith, ${ }^{35}$ D. I. Sober, ${ }^{6}$ D. Sokhan, ${ }^{35}$ S. Stepanyan, ${ }^{34}$ S. S. Stepanyan, ${ }^{24}$ S. Strauch, ${ }^{14,33}$ W. Tang, ${ }^{28}$ M. Ungaro, ${ }^{9,34}$ A. V. Vlassov, ${ }^{22}$ H. Voskanyan, ${ }^{40}$ E. Voutier, ${ }^{25}$ N. K. Walford, ${ }^{6}$ D. Watts, ${ }^{10}$ X. Wei, ${ }^{34}$ L. B. Weinstein, ${ }^{29}$ M. H. Wood, ${ }^{4,33}$

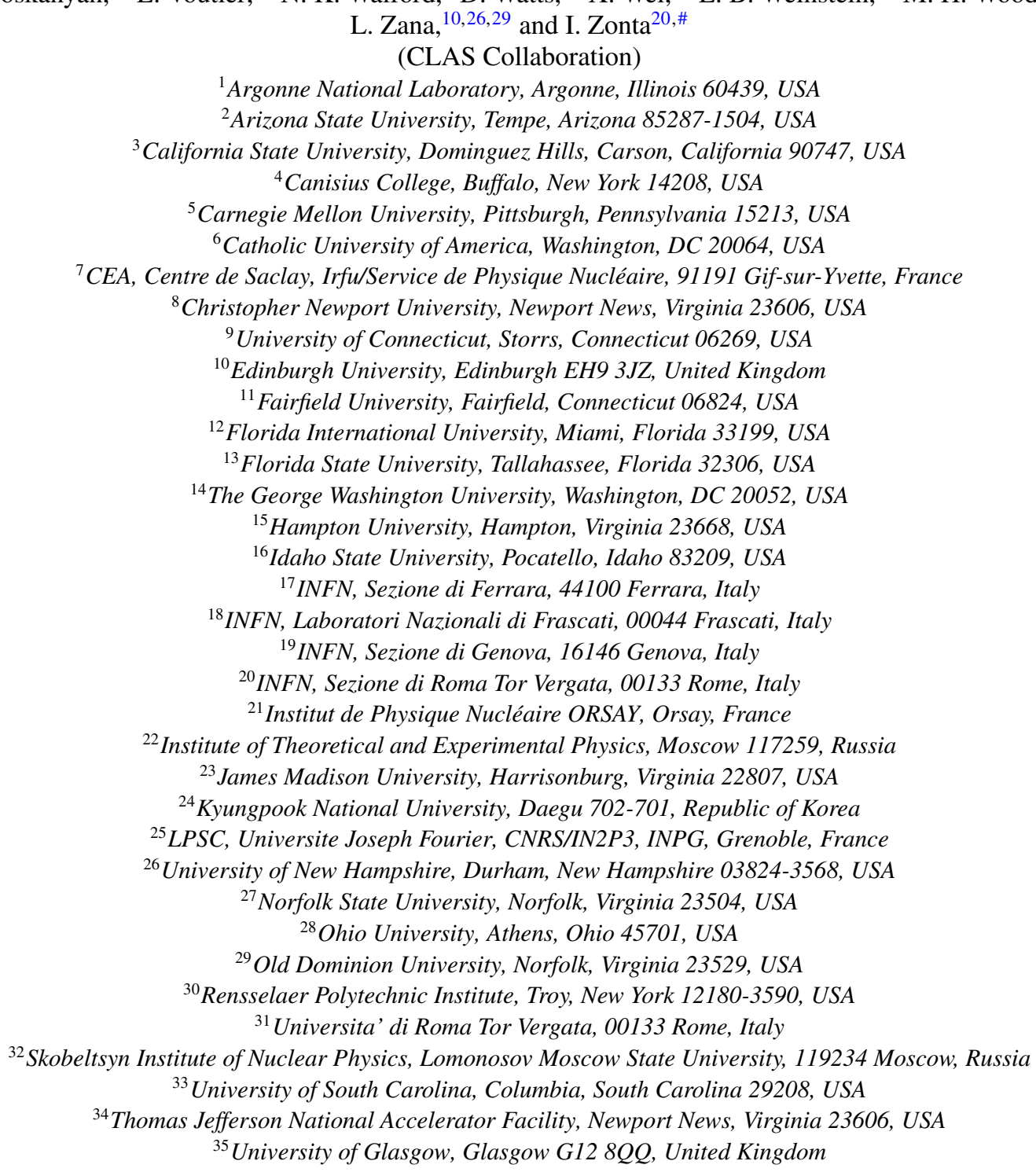




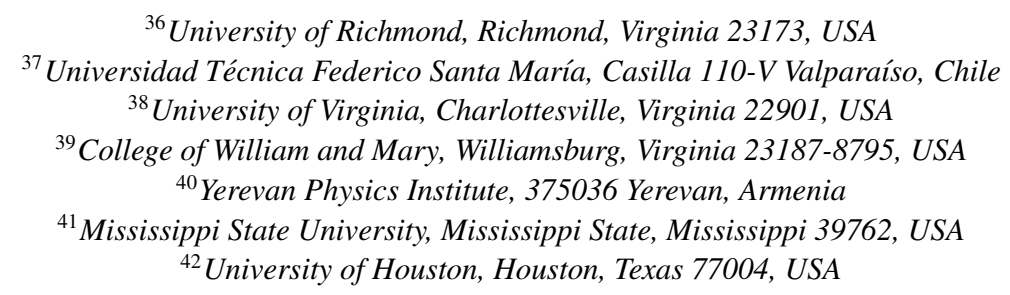

(Received 13 February 2014; published 24 April 2014; corrected 5 November 2014)

\begin{abstract}
Background: Much less is known about neutron structure than that of the proton due to the absence of free neutron targets. Neutron information is usually extracted from data on nuclear targets such as deuterium, requiring corrections for nuclear binding and nucleon off-shell effects. These corrections are model dependent and have significant uncertainties, especially for large values of the Bjorken scaling variable $x$. As a consequence, the same data can lead to different conclusions, for example, about the behavior of the $d$ quark distribution in the proton at large $x$.

Purpose: The Barely Off-shell Nucleon Structure experiment at Jefferson Lab measured the inelastic electrondeuteron scattering cross section, tagging spectator protons in coincidence with the scattered electrons. This method reduces nuclear binding uncertainties significantly and has allowed for the first time a (nearly) modelindependent extraction of the neutron structure function $F_{2}\left(x, Q^{2}\right)$ in the resonance and deep-inelastic regions.

Method: A novel compact radial time projection chamber was built to detect protons with momentum between 70 and $150 \mathrm{MeV} / c$ and over a nearly $4 \pi$ angular range. For the extraction of the free-neutron structure function $F_{2}^{n}$, spectator protons at backward angles $\left(>100^{\circ}\right.$ relative to the momentum transfer) and with momenta below $100 \mathrm{MeV} / c$ were selected, ensuring that the scattering took place on a nearly free neutron. The scattered electrons were detected with Jefferson Lab's CLAS spectrometer, with data taken at beam energies near 2, 4, and $5 \mathrm{GeV}$. Results: The extracted neutron structure function $F_{2}^{n}$ and its ratio to the inclusive deuteron structure function $F_{2}^{d}$ are presented in both the resonance and the deep-inelastic regions for momentum transfer squared $Q^{2}$ between 0.7 and $5 \mathrm{GeV}^{2} / c^{2}$, invariant mass $W$ between 1 and $2.7 \mathrm{GeV} / c^{2}$, and Bjorken $x$ between 0.25 and 0.6 (in the deep-inelastic scattering region). The dependence of the semi-inclusive cross section on the spectator proton momentum and angle is investigated, and tests of the spectator mechanism for different kinematics are performed. Conclusions: Our data set on the structure function ratio $F_{2}^{n} / F_{2}^{d}$ can be used to study neutron resonance excitations, test quark-hadron duality in the neutron, develop more precise parametrizations of structure functions, and investigate binding effects (including possible mechanisms for the nuclear EMC effect) and provide a first glimpse of the asymptotic behavior of $d / u$ at $x \rightarrow 1$.
\end{abstract}

\section{DOI: 10.1103/PhysRevC.89.045206}

\section{INTRODUCTION}

The advent of high-luminosity beams at modern accelerator facilities such as the Continuous Electron Accelerator Facility (CEBAF) at Jefferson Lab has opened the way for dedicated programs of nucleon structure measurements with unprecedented precision. The data have allowed phenomena such as quark-hadron duality and the transition to scaling in transverse and longitudinal nucleon structure functions to be accurately verified, as well as precision studies to be conducted of the flavor and spin structure of the proton in kinematic regions

*Current address: University of Virginia, Charlottesville, VA 22901.

${ }^{\dagger}$ Corresponding author: skuhn@ odu.edu

${ }^{\ddagger}$ Current address: Institut de Physique Nucléaire ORSAY, Orsay, France.

${ }^{\S}$ Current address: Universidad Técnica Federico Santa María, Casilla 110-V Valparaíso, Chile.

"Current address: Old Dominion University, Norfolk, VA 23529.

"Current address: Thomas Jefferson National Accelerator Facility, Newport News, VA 23606.

\#Current address: Universita' di Roma Tor Vergata, 00133 Rome, Italy.
PACS number(s): 13.60.Hb, 14.20.Dh, 24.85.+p, 25.30.Fj

previously inaccessible (see, e.g., Refs. [1-3] and references therein).

In particular, the region of large parton (quark) momentum fraction $(x \gtrsim 0.5)$, which is experimentally challenging because of the small cross sections involved, has seen a resurgence of interest in recent years [4,5], especially at Jefferson Lab with its unique access to large $x$. Part of this interest has been the promise to resolve decades-long questions about parton distribution functions (PDFs) at large $x$, such as the behavior of the unpolarized $d / u$ or polarized $\Delta d / d$ ratios in the $x \rightarrow 1$ limit. At large four-momentum transfer squared, $Q^{2} \gg 1 \mathrm{GeV}^{2} / c^{2}$, these offer relatively clean probes of the strong interaction dynamics of valence quarks in the nucleon. To access information on $d$ quarks, and in particular these ratios, one needs electron-scattering data from both proton and neutron targets. However, while experiments have been able to map out in great detail the characteristics of the proton at large $x$, determining the corresponding structure of the neutron has proved to be much more difficult.

At lower values of $Q^{2}$ (of order $1 \mathrm{GeV}^{2} / c^{2}$ ), the large- $x$ region is dominated by nucleon resonances, among which the $\Delta(1232)$ is the lowest-mass excitation. A fundamental question here is whether the ratio $\sigma_{n} / \sigma_{p}$ of neutron- to proton-inclusive electron-scattering cross sections for the 
$N \rightarrow \Delta(1232)$ transition is unity, as would be expected for a pure isovector transition $(\Delta I=1)$. Existing deuteron electroproduction data [6-8] indicate that the isotensor $(\Delta I=2)$ contribution is small but non-negligible. Similarly, comparing inclusive cross sections on the neutron with those on the proton for the higher-lying (overlapping) resonance excitations can provide constraints on the isospin structure of the resonant and nonresonant contributions to the total cross section. Finally, neutron structure functions in the resonance region are needed to conclusively test Bloom-Gilman duality [9] in the neutron.

The absence of free neutron targets has meant that, in practice, light nuclei such as the deuteron and ${ }^{3} \mathrm{He}$ are routinely used as effective neutron targets. In regions of kinematics where most of the neutron's momentum is carried by a single valence quark, or where the spectrum is dominated by resonances, different choices for models of nuclear corrections can lead to significant uncertainties in the neutron cross sections [10-15]. Consequently, our ability to determine unambiguously the isospin structure of the nucleon PDFs, as well as the spectrum of the excited states of the nucleon, has been severely limited. For example, in the nucleon resonance region there are large uncertainties in the neutron to $N^{*}$ transition helicity amplitudes extracted from deuteron measurements, while in the deep-inelastic scattering (DIS) region the $d$-quark PDF is poorly determined beyond $x \sim 0.6$. Aside from the intrinsic value of such knowledge, a practical ramification is that the large- $x$ PDF uncertainties can in some cases propagate to influence production rates of particles, including those predicted beyond the standard model, at high-energy colliders such as the Large Hadron Collider [16,17].

To move beyond this impasse, it has been suggested [18-21] that one can minimize the nuclear model uncertainties by selecting (or "tagging") final states in the electron-deuteron scattering process in which the proton is produced with small momentum in the backward hemisphere relative to the momentum transfer. This minimizes the probability of rescattering of the "spectator" proton with the rest of the hadronic debris, thereby ensuring that the reaction took place on a neutron close to its mass shell [22,23].

The first direct extraction of inclusive scattering data on a nearly free neutron using this spectator tagging technique was performed with the Barely Off-shell Nucleon Structure (BONuS) experiment at Jefferson Lab, which ran in 2005 in Hall B using CLAS and a novel radial time projection chamber (RTPC) capable of detecting protons with momenta down to $70 \mathrm{MeV} / c$. In a first report [24], a representative sample of the $\mathrm{BONuS}$ neutron spectra was presented, allowing a first glimpse into the inclusive neutron excited mass spectrum and the neutron $F_{2}^{n}$ structure function at large $x$, essentially free of nuclear correction uncertainties. In this paper we present the full $\mathrm{BONuS}$ data sample. These data cover a large kinematic range, from the quasielastic peak to the region of final-state hadron masses $W \approx 2.7 \mathrm{GeV} / c^{2}$ and $Q^{2}$ from 0.7 to $5 \mathrm{GeV}^{2} / c^{2}$.

In Sec. II we review the basic formulas for describing spectator proton tagging in semi-inclusive scattering from the deuteron within the impulse approximation (IA) and discuss various corrections to the IA due to final-state interactions, nucleon off-shellness, and other effects. An overview of the experimental setup is presented in Sec. III, where we outline the novel features of the BONuS RTPC. Details of the data analysis are given in Sec. IV, which describes the event selection and background subtraction and two different methods of analysis. The results of the experiment are presented in Sec. V. We present results both for the "spectator limit" (slow, backward protons), which can be used to constrain models of neutron structure with minimal nuclear binding uncertainties, and for kinematics in which nuclear and final-state interaction effects are enhanced (forward and higher-momentum protons). Our analysis allows us to identify kinematic regions in which the spectator approximation can be used for extracting the free neutron structure function. Finally, in Sec. VI we summarize our findings and discuss future extensions of the spectator tagging technique planned at the energy-upgraded $12-\mathrm{GeV}$ Jefferson Lab facility.

\section{PHYSICS OVERVIEW}

In this section we review the physics motivation for the BONuS experiment and the formalism employed to analyze semi-inclusive scattering from the deuteron with a tagged spectator proton. We discuss the accuracy of the nuclear IA used to extract the neutron structure function from the semiinclusive cross section and examine various corrections to the IA from final-state interactions and nucleon off-shell effects.

\section{A. Motivation}

There are a number of reasons why knowledge of the free neutron structure functions is vital for our understanding of the quark structure of the nucleon and nonperturbative QCD more generally. In the nucleon resonance region, an accurate determination of neutron structure functions is needed for the extraction of the full isospin dependence of the resonant and nonresonant contributions to the inclusive neutron cross section. Knowledge of the neutron resonance structure is also needed for the model-independent verification of Bloom-Gilman duality in the neutron $[3,9,25,26]$ and for understanding the transition between the resonance and deepinelastic regions. While existing model-dependent studies [27] suggest a common origin of duality for the neutron and proton, proof of this requires neutron resonance data that are free of nuclear model assumptions.

Unfortunately, the absence of high-density, free neutron targets has usually forced neutron structure to be extracted from inclusive scattering experiments on nuclear targets, such as the deuteron. Such extractions, however, necessarily involve model-dependent methods to account for nuclear effects in the deuteron [27]. The extraction of the neutron structure function in the resonance region from inclusive nuclear data is particularly challenging because of Fermi smearing, which acts to reduce the distinctiveness of the resonance peaks from the nonresonant background [28].

Of course, definitive tests of quark-hadron duality must involve data from both the resonance and the DIS regions. For the latter, the parton model allows the structure of the nucleon to be characterized in terms of the nucleon's valence $u$ - and $d$-quark momentum distributions. Following many years of DIS and other high-energy scattering experiments, a detailed picture has emerged of the structure of the nucleon at intermediate and small values of Bjorken $x$. The abundance 


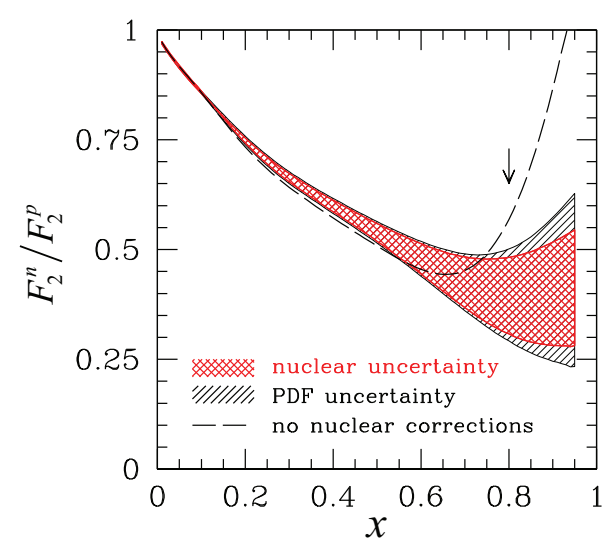

FIG. 1. (Color online) Ratio of inclusive neutron-to-proton structure functions $F_{2}^{n} / F_{2}^{p}$ from the CJ global PDF analysis [13]. The shaded bands illustrate the range of possible values for the ratio from nuclear corrections and experimental uncertainties. The vertical arrow indicates the edge of the region in $x$ where the ratio is constrained by data $(x \lesssim 0.8)$.

of high-precision proton structure function $\left(F_{2}^{p}\right)$ data has, due to the preferential coupling of the photon to $u$ quarks compared with $d$ quarks in the proton, allowed an accurate determination of the $u$-quark PDF at both small and large values of $x$.

The corresponding $d$-quark distribution could be similarly constrained by neutron structure function $\left(F_{2}^{n}\right)$ data and the $d / u$ ratio extracted, at leading order in the strong coupling constant and for $x \gtrsim 0.5$, via

$$
\frac{d}{u} \approx \frac{4 F_{2}^{n} / F_{2}^{p}-1}{4-F_{2}^{n} / F_{2}^{p}}
$$

where the approximation neglects strange and heavier quarks. At high values of $x$ (where large nucleon momenta contribute significantly in nuclei) the uncertainties associated with the nuclear corrections propagate to the extracted neutron structure functions and hence to the $F_{2}^{n} / F_{2}^{p}$ ratio [10-15]. The results for $F_{2}^{n} / F_{2}^{p}$ from a recent global fit by the CTEQ-Jefferson Lab (CJ) Collaboration [13] are illustrated in Fig. 1, showing the uncertainties from both nuclear corrections and experiment. Beyond $x \approx 0.5$ the current data not only prevent us from understanding the basic nonperturbative dynamics responsible for the behavior of $d / u$ in the $x \rightarrow 1$ limit, for which predictions range from 0 to $\approx 0.5[4,11]$, but can also impact our ability to reliably compute QCD cross sections in high-energy collider experiments which have sensitivity to the $d$-quark PDF [17].

Measurement of the free neutron structure function would also allow for a model-independent determination of the size of the nuclear correction in the deuteron through the construction of the $F_{2}^{d} /\left(F_{2}^{p}+F_{2}^{n}\right)$ ratio. This would provide data that could discriminate between various detailed models of nuclear effects in the deuteron [18,29-33], thereby solving the decades-long question about the magnitude of the nuclear EMC effect in the deuteron. Finally, reliable parametrizations for $F_{2}^{n}$ are needed to extract ratios of nuclear to nucleon structure functions from inclusive measurements on nuclear targets and on spin structure functions from polarization asymmetries in inclusive scattering.

\section{B. Spectator tagging}

Because the deuteron is a weakly bound system with binding energy $\epsilon_{d}=-2.2 \mathrm{MeV}$ (only about $0.1 \%$ of the deuteron mass), on average the deuteron structure function may be reasonably well approximated by a sum of free proton and neutron structure functions. At large values of $x$, however, the deuteron structure functions receive increasingly greater contributions from nucleons carrying a larger fraction of the deuteron's momentum. These contributions are sensitive to the details of the high-momentum tails of the deuteron wave function, which are not as well constrained by nucleonnucleon scattering data as the low-momentum components. Consequently, in the high- $x$ region there is a more significant dependence on the model for the smearing of the nucleon structure due to binding and Fermi motion effects, as well as to possible modifications of nucleon structure when the nucleon is off its mass shell.

The nuclear model uncertainties in the extraction of the neutron structure function from inclusive electron-deuteron scattering data can be significantly reduced by detecting low-momentum protons produced at backward kinematics, relative to the momentum transfer, in coincidence with the scattered electron,

$$
e+d \rightarrow e+p_{s}+X .
$$

The restriction to low momenta ensures that the scattering takes place on a nearly on-shell neutron [19-21], while tagging backward-moving spectator protons $\left(p_{s}\right)$ minimizes final-state interaction effects $[22,23]$.

The cross section for the semi-inclusive electroproduction of a proton with four-momentum $p_{s}^{\mu}=\left(E_{s}, \boldsymbol{p}_{s}\right)$ can be written in the deuteron rest frame as $[21,23]$

$$
\begin{aligned}
& \frac{d \sigma}{d x d} Q^{2} d^{3} \boldsymbol{p}_{s} / E_{s} \\
& =\frac{4 \pi \alpha_{\mathrm{em}}^{2}}{x Q^{4}}\left(1-y-\frac{x^{2} y^{2} M^{2}}{Q^{2}}\right) \\
& \quad \times\left[F_{L}^{d}+\left(\frac{Q^{2}}{2 \boldsymbol{q}^{2}}+\tan ^{2} \frac{\theta}{2}\right) \frac{v}{M} F_{T}^{d}\right. \\
& \left.\quad+\sqrt{\frac{Q^{2}}{2 \boldsymbol{q}^{2}}+\tan ^{2} \frac{\theta}{2}} \cos \phi F_{T L}^{d}+\cos 2 \phi F_{T T}^{d}\right],
\end{aligned}
$$

where $\alpha_{\mathrm{em}}$ is the electromagnetic fine structure constant and $E_{s}=\sqrt{M^{2}+p_{s}^{2}}$ and $M$ are the energy and mass, respectively, of the spectator proton produced at an azimuthal angle $\phi$ around the $z$ axis (defined along the $\boldsymbol{q}$ direction). The fourmomentum transfer to the deuteron is given by $q^{\mu}=(v, \boldsymbol{q})$, with $Q^{2} \equiv-q^{2}$ and $x=Q^{2} / 2 M v$ the usual Bjorken scaling variable evaluated in the target rest frame. The variable $y=v / E_{e}$ denotes the fractional loss of the electron energy $E_{e}$, and $\theta$ is the electron-scattering angle.

The semi-inclusive deuteron structure functions $F_{L}^{d}, F_{T}^{d}$, $F_{T L}^{d}$, and $F_{T T}^{d}$ depend on the variables $x$ and $Q^{2}$, the light-cone momentum fraction of the spectator proton $\alpha_{s}=\left(E_{s}-p_{s}^{z}\right) / M$, and the spectator proton transverse momentum $p_{s}^{\perp}$. In terms of the angle between the outgoing spectator proton and the direction of $\boldsymbol{q}$, the longitudinal and transverse 


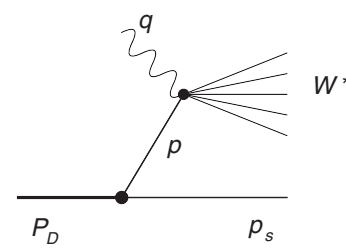

(a)

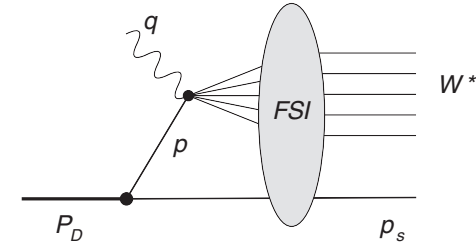

(b)
FIG. 2. Semi-inclusive scattering from a deuteron with detection of a spectator proton, $p_{s}$, within the framework of (a) the nuclear IA and (b) including the effects of final-state interactions.

spectator momenta are given by $p_{s}^{z}=\left|\boldsymbol{p}_{s}\right| \cos \theta_{p q}$ and $p_{s}^{\perp}=$ $\left|\boldsymbol{p}_{s}\right| \sin \theta_{p q}$, respectively. Integrating over the azimuthal angle $\phi$, the terms proportional to $F_{T L}^{d}$ and $F_{T T}^{d}$ vanish, and the cross section of Eq. (3) becomes proportional to the familiar combination of semi-inclusive (SI) structure functions $(2 v / M) \tan ^{2}(\theta / 2) F_{1}^{d(\mathrm{SI})}+F_{2}^{d(\mathrm{SI})}$, where

$$
\begin{aligned}
& F_{1}^{d(\mathrm{SI})}=\frac{1}{2} F_{T}^{d}, \\
& F_{2}^{d(\mathrm{SI})}=F_{L}^{d}+\frac{x}{\rho^{2}} F_{T}^{d},
\end{aligned}
$$

with $\rho^{2}=1+4 M^{2} x^{2} / Q^{2}$. The semi-inclusive structure functions $F_{1,2}^{d(\mathrm{SI})}$ are then related to the inclusive deuteron structure functions $F_{1,2}^{d}$ simply by integrating over the spectator proton momentum $\boldsymbol{p}_{s}$.

In the nuclear IA, illustrated in Fig. 2(a), the virtual photon scatters incoherently from the bound neutron with four-momentum $p^{\mu}$, where $p^{\mu}+p_{s}^{\mu}=P_{d}^{\mu}=\left(M_{d}, \mathbf{0}\right)$ in the deuteron rest frame, with $M_{d}$ the deuteron mass. In this case the semi-inclusive deuteron structure functions can be written as products of the structure functions of the bound neutron and the nuclear spectral function $S\left(\alpha_{s}, p_{s}^{\perp}\right)$ [21,23],

$$
\begin{aligned}
F_{1}^{d(\mathrm{SI})}\left(x, Q^{2}, \alpha_{s}, p_{s}^{\perp}\right) \approx & S\left(\alpha_{s}, p_{s}^{\perp}\right)\left[F_{1}^{n, \text { eff }}\left(x^{*}, Q^{2}, \alpha, p^{\perp}\right)\right. \\
& \left.+\frac{p^{\perp 2}}{2 p q} F_{2}^{n, \text { eff }}\left(x^{*}, Q^{2}, \alpha, p^{\perp}\right)\right], \quad(5 \mathrm{a}) \\
F_{2}^{d(\mathrm{SI})}\left(x, Q^{2}, \alpha_{s}, p_{\perp s}\right) \approx & S\left(\alpha_{s}, p_{s}^{\perp}\right) \frac{M v}{p q}\left\{\left(1+\sqrt{1-\frac{Q^{2}}{2 \boldsymbol{q}^{2}}}\right)^{2}\right. \\
& \left.\times\left[\alpha+\frac{2 p q}{(v+|\boldsymbol{q}|) M_{d}}\right]^{2}+\frac{Q^{2}}{2 \boldsymbol{q}^{2}} \frac{p_{s}^{\perp 2}}{M^{2}}\right\} \\
& \times F_{2}^{n, \text { eff }}\left(x^{*}, Q^{2}, \alpha, p^{\perp}\right),
\end{aligned}
$$

where $F_{1,2}^{n, \text { eff }}$ are the bound or "effective" neutron structure functions. In the on-shell limit, the bound neutron structure functions reduce to the free neutron structure functions, $F_{1,2}^{n, \text { eff }} \rightarrow F_{1,2}^{n}$, but in general are functions of the off-shell neutron's invariant Bjorken variable

$$
x^{*}=\frac{Q^{2}}{2 p q} \approx \frac{x}{\alpha},
$$

the struck neutron's light-cone momentum fraction $\alpha=2-$ $\alpha_{s}$, and its transverse momentum $\boldsymbol{p}^{\perp}=-\boldsymbol{p}_{s}^{\perp}$. Alternatively, one can also express $F_{1,2}^{n, \text { eff }}$ as a function of the final-state invariant mass squared,

$$
W^{* 2}=(p+q)^{2}=p^{2}+\frac{Q^{2}\left(1-x^{*}\right)}{x^{*}},
$$

where $p^{2}=\left(M_{d}-E_{s}\right)^{2}-p^{2}$ is the invariant mass squared of the off-shell nucleon. Note that in the on-shell limit, the struck nucleon's Bjorken variable $x^{*} \rightarrow x$, while $W^{* 2}$ reduces to the invariant mass squared $W^{2}=M^{2}+Q^{2}(1-x) / x$ for a free nucleon at rest.

The nuclear spectral function $S$ describes the probability of finding an off-shell neutron in the deuteron with momentum $\left(\alpha, p^{\perp}\right)$ and an on-shell proton with momentum $\left(\alpha_{s}, p_{s}^{\perp}\right)$. It is determined by the square of the deuteron wave function $\left|\psi_{d}(p)\right|^{2}$ and kinematic factors that depend on the framework used to compute the nuclear structure function. These factors coincide in the limit where both nucleons are on shell, but differ in the off-shell behavior [20,21]. The expressions in Eqs. (5) for the semi-inclusive structure functions can be used to extract the free neutron $F_{1,2}^{n}$ structure functions in the limit $\alpha_{s} \rightarrow 1$ and $p_{s}^{\perp} \rightarrow 0$. Of course, the experimental data will only be available for some minimum value of $p_{s}^{\perp}$, which will introduce some uncertainty into the on-shell extrapolation, as discussed in the following sections.

While uncertainties in the nucleon-nucleon interaction at short distances lead to significant dependence of the inclusive deuteron structure function on the deuteron wave function for $x \gtrsim 0.6$ [13], restricting the spectator proton momenta to $\left|\boldsymbol{p}_{s}\right| \lesssim 100 \mathrm{MeV} / \mathrm{c}$ renders these negligible. Furthermore, comparisons of spectral functions computed within the instantform and light-front formulations suggest [20] that at these momenta and $\alpha \lesssim 1.1$ the model dependence of the spectral function is at the few percent level.

\section{Beyond the impulse approximation}

\section{Final-state interactions}

Although Eqs. (5) describe semi-inclusive proton production in the nuclear IA, interactions between the recoil proton and the hadronic debris of the scattered neutron, illustrated in Fig. 2(b), can, in principle, distort the momentum distribution of the detected protons. Microscopic calculations of the finalstate interaction (FSI) effects within hadronization models and the distorted wave IA suggest strong suppression of FSIs at backward spectator proton angles $\theta_{p q}$ relative to the photon direction $[22,23,34]$.

The main uncertainty in estimating the role of FSIs is the spectator proton-hadronic debris $(X)$ scattering cross section $\sigma_{p X}$. Frankfurt et al. [34] estimated this from the ${ }^{2} \mathrm{H}\left(e, e^{\prime} p\right) n$ breakup reaction at high energies using data on soft neutron production in muon DIS from heavy nuclei [35]. At backward angles FSIs were found to contribute less than $5 \%$ to the cross section for $p_{s}^{\perp}<100 \mathrm{MeV} / c$ and $\alpha_{s}<1.5$.

In the hadronization model of Ciofi degli Atti et al. [22] the rescattering cross section $\sigma_{p X}$ was derived from a color flux tube picture and found to grow logarithmically with 
time. Including the effects of color string breaking and gluon bremsstrahlung, the resulting FSI corrections were again small in the backward hemisphere, amounting to $\lesssim 5 \%$ for spectator angles $\theta_{p q}>120^{\circ}$ and $\left|\boldsymbol{p}_{s}\right| \lesssim 100 \mathrm{MeV} / c$. For larger momenta, $\left|\boldsymbol{p}_{s}\right| \approx 200 \mathrm{MeV} / c$, FSIs enhance the spectral function by $\approx 20 \%$ at backward angles. FSIs are most pronounced in perpendicular kinematics, $\theta_{p q} \sim 90^{\circ}$, where they can be used as a tool to study the process of hadronization in nuclei. Models such as that of Ciofi degli Atti et al. [22] predict that in this angular region, FSI can lead to either a suppression (for $\left|\boldsymbol{p}_{s}\right| \leqslant 200 \mathrm{MeV} / c$ ) or a significant enhancement (for $\left|\boldsymbol{p}_{s}\right| \geqslant 400 \mathrm{MeV} / c$ ) of the cross section. In all existing models, however, it is clear that FSIs can be minimized to $\lesssim 5 \%$ by restricting proton momenta to $\left|\boldsymbol{p}_{s}\right| \lesssim 100 \mathrm{MeV} / c$ and spectator angles to $\theta_{p q} \gtrsim 100^{\circ}$, which serves as a guide for the kinematic cuts utilized in the BONuS experiment.

\section{Target fragmentation}

Backward kinematics also suppresses hadronization of low-momentum protons produced from the debris of the struck neutron [19,36,37]. Although a potentially important contribution in the forward hemisphere (current fragmentation region), direct fragmentation into protons was found by Simula [19] to be negligible for $\theta_{p q} \gtrsim 90^{\circ}$ even for large momenta $p_{s}$.

\section{Nucleon off-shell effects}

The dependence of the bound neutron structure functions on the neutron's off-shell mass squared $p^{2} \approx M^{2}+2 M \epsilon_{d}-2 p_{s}^{2}$ can introduce additional deviations of the extracted structure functions in Eqs. (5) from their on-shell values. However, the restriction to low-momentum protons guarantees that the neutron's virtuality $M^{2}-p^{2}$ does not exceed $\approx 13 \mathrm{MeV}^{2} / c^{2}$ for $p_{s}=100 \mathrm{MeV} / c$ and $\approx 7 \mathrm{MeV}^{2} / c^{2}$ for $p_{s}=70 \mathrm{MeV} / c$, the lower acceptance limit of the BONuS detector.

Determining the effect of the nucleon's virtuality on its structure from first principles is extremely challenging and, in fact, cannot be rigorously defined independently of the nucleon's environment. The off-shell effects have been estimated within several models of the nucleon, including dynamical quark-diquark models $[29,30]$ and effective models in which the bound nucleon structure functions are evaluated at shifted kinematics [38,39].

In the covariant quark-(spectator) diquark model of Melnitchouk et al. [29], scattering from a bound nucleon is described in terms of relativistic vertex functions that parametrize the nucleon-quark-(spectator) diquark interaction, with the vertex functions constrained by inclusive $F_{2}^{p}$ and $F_{2}^{d}$ data. The off-shell effects at low $p_{s}$ are small, as expected, and increase at higher momenta. For $p_{s}<100 \mathrm{MeV} / c$, the correction is essentially zero at $x \approx 0.3$, and does not exceed $\approx 1 \%$ at larger $x$.

A similar model introduced by Gross and Liuti [39] describes scattering from an off-shell nucleon in terms of a relativistic quark spectral function, with the bound nucleon structure function evaluated at a shifted value of $x$ that depends on the mass of the diquark, the bound nucleon momentum, and the binding energy. The effects are again small at low spectator proton momenta, $\lesssim 2 \%$ for $p_{s}<100 \mathrm{MeV} / c$, increasing to around $5 \%$ for $p_{s}=200 \mathrm{MeV} / c$.

Simply on the basis of kinematics, Heller and Thomas [38] also estimated the role of nucleon off-shellness within an instant form approach, in which the bound nucleon structure function was evaluated at a shifted energy transfer that is correlated with the degree to which the nucleon is off its energy shell. The off-shell modifications here were found to be $\lesssim 1 \%$ for low spectator momenta $p_{s} \approx 100 \mathrm{MeV} / c$.

In all cases considered, therefore, the effects of the neutron's off-shellness play only a very minor role as long as spectator proton momenta are restricted to values $p_{s}<100 \mathrm{MeV} / c$. At larger $p_{s}$ the off-shell effects can be studied in conjunction with data from earlier experiments [40], which measured spectator proton spectra over the range $280<p_{s}<700 \mathrm{MeV} / c$, as a means of probing the medium modifications of the nucleon's quark structure.

\section{EXPERIMENTAL SETUP}

The BONuS experiment was conducted in Hall B of the Thomas Jefferson National Accelerator Facility (TJNAF or Jefferson Lab). Electrons from the CEBAF beam were scattered off a deuteron target and detected by CLAS. The spectator protons were detected with an RTPC designed specially for this experiment.

CEBAF is a superconducting radio-frequency accelerator facility capable of delivering continuous polarized electron beams with energies up to $6 \mathrm{GeV}$. (It is presently being upgraded for up to $12-\mathrm{GeV}$ beam energy.) During the BONuS experiment, beam energies of approximately $1.1,2.14,4.23$, and $5.27 \mathrm{GeV}$ with beam currents from $2 \mathrm{nA}$ up to $55 \mathrm{nA}$ were employed.

\section{A. CLAS}

The Hall B end station houses the CEBAF Large Acceptance Spectrometer (CLAS). CLAS can detect particles for $\theta$ angles $8^{\circ}-142^{\circ}$ and for approximately $80 \%$ of $2 \pi$ in $\phi$. It employs a toroidal magnetic field of up to $2 \mathrm{~T}$ produced by six superconducting coils. CLAS consists of several layers of particle detectors, each separated into six azimuthal sectors by the torus magnet coils.

(i) Drift chambers (DCs), which determine chargedparticle trajectories. They are capable of a momentum resolution of $\delta p / p \leqslant 0.5 \%$ and angular track resolution of $\delta \theta \leqslant 1 \mathrm{mrad}, \delta \phi \leqslant 5 \mathrm{mrad}$ for $1 \mathrm{GeV} / c$ particles [41].

(ii) Cherenkov counters (CCs) for electron-pion separation (used in the trigger). CLAS CCs are capable of distinguishing pions and electrons up to momenta of approximately $2.8 \mathrm{GeV} / c$ [42].

(iii) Scintillation counters (SCs) for time-of-flight (TOF) measurements. The counters cover the $\theta$ range between $8^{\circ}$ and $142^{\circ}$ and the entire active range in $\phi$ (for a total area of $206 \mathrm{~m}^{2}$ ) [43]. The time resolution of the system is between 70 ps (for the shortest counters) and 165 ps (for the longest counters). 


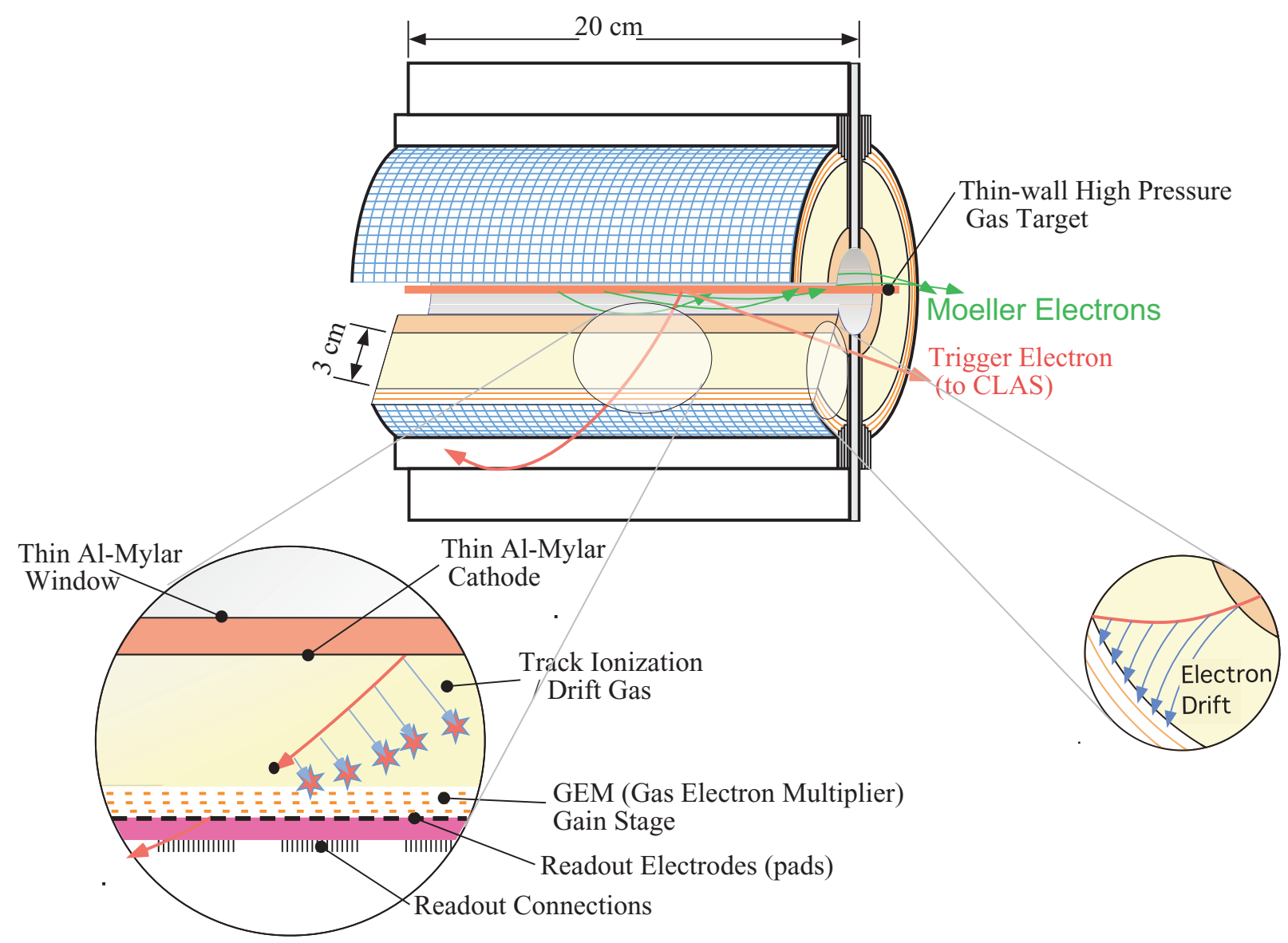

FIG. 3. (Color online) Schematics of the BONuS RTPC. See text for details.

(iv) Electromagnetic calorimeters (ECs) to identify electrons and to detect neutral particles like photons and neutrons. The ECs are used to trigger on electrons at energies above $0.5 \mathrm{GeV}$. The sampling fraction is approximately 0.3 for electrons of $3 \mathrm{GeV}$ and greater, and for smaller energies, there is a monotonic decrease to about 0.25 for electrons of $0.5 \mathrm{GeV}$ [44]. The average rms resolution is $2.3 \mathrm{~cm}$ for electron showers with more than $0.5 \mathrm{GeV}$ of energy deposited in the scintillator. The timing resolution of the EC for electrons averages to 200 ps over the entire detector.

All detectors listed above are standard CLAS equipment and have been in Hall B for over a decade. CLAS is described in detail in Ref. [45]. They were complemented by a dedicated RTPC utilizing gas electron multipliers (GEMs) that was built specifically for this experiment (see below). It was designed to detect heavily ionizing, slow moving protons that cannot travel far from the target.

\section{B. Radial time projection chamber}

To identify events in which a proton is a mere "spectator" to the electron-neutron collision, we needed to select events in which the detected proton is moving backwards with low momentum (around or below $0.1 \mathrm{GeV} / c$ ). To register such protons, we needed a detector that provides good coverage in the backward hemisphere (with respect to the direction of the electron beam) and is close enough to the target to be able to detect these heavily ionizing low-energy protons before they get stopped. An RTPC [46] utilizing GEMs was constructed for this experiment to fulfill these requirements (see Fig. 3). The RTPC was surrounded by a solenoid magnet, run at 3.5 and 4.7 $\mathrm{T}$, that served to analyze proton momenta and, in addition, to deflect Moeller electron trajectories, making them stay clear of all sensitive detector volumes.

The capability of time projection chambers (TPCs) to provide a complete $3 \mathrm{D}$ picture of particle trajectories in the detector volume, as well as particle identification through specific energy loss, $d E / d x$, combined with the low mass density of this kind of detector, made it a natural choice for our purposes. The BONuS RTPC utilizes gas for its sensitive volume to reduce the mass density the protons have to traverse. The more common axial TPC would not have been a good choice for the following reasons.

(i) The solenoid magnet length is less than its diameter, and so it does not have magnetic field lines parallel to each other over a reasonable length.

(ii) Detecting forward-moving high-momentum particles with CLAS requires minimizing the end-cap density, the region where a lot of equipment is normally situated in axial TPCs. 


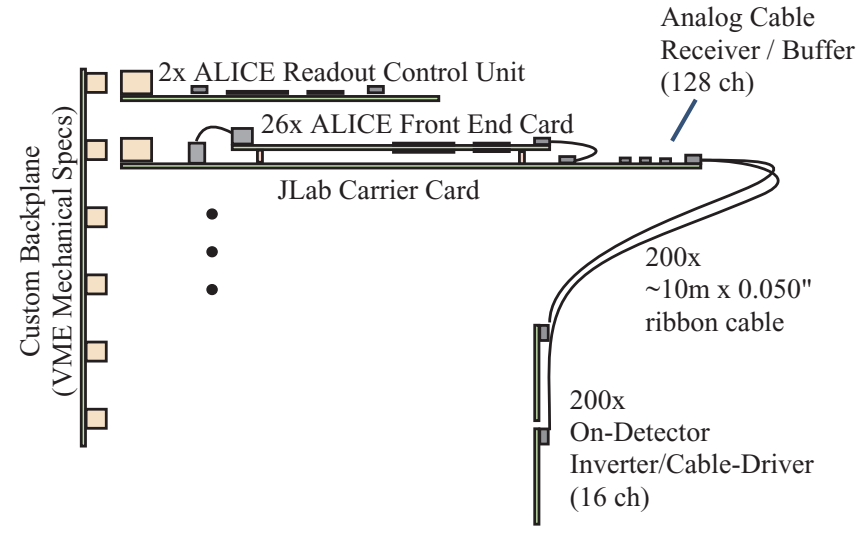

FIG. 4. (Color online) BONuS data readout scheme.

(iii) The RTPC configuration made it easier to stay clear of the Moeller electrons.

RTPCs, in which electrons drift radially outwards from the cylindrical central cathode to the anode located on a concentric cylinder, have been previously used, e.g., by the STAR [47] and CERES [48] collaborations. In this configuration, the electric and magnetic fields are no longer parallel, which leads to complex electron drift trajectories. In addition, curved readout pad planes are required. For these reasons RTPCs have a more complex structure.

Because the charge collected at the readout pads is proportional to the energy loss of the particle, the signal amplitude at the pads as a function of time provides information on the specific energy loss of the particle. A particle's momentum and charge can be found from the curvature of its trajectory in the magnetic field; hence, the particle can be identified. This requires a quasicontinuous readout of amplitude information from the pads, generating a potentially large data flow. We designed the BONuS RTPC around custom integrated circuits built for the large TPC used in the ALICE heavy-ion experiment at CERN [49,50] (see Fig. 4).

Figure 3 shows the BONuS RTPC with the integrated $7 \mathrm{~atm}$ deuterium gas target on its axis. The target has a fiducial length of $17 \mathrm{~cm}$ (visible by the RTPC) and inner diameter of $0.6 \mathrm{~cm}$ with 50- $\mu \mathrm{m}$ Kapton walls. The detector surrounds the target at close distance with the center of the RTPC moved $25 \mathrm{~mm}$ with respect to the target center for better coverage of the backwards hemisphere, where spectator protons are expected. Upon exiting the target and traversing a buffer volume filled with $1 \mathrm{~atm}$ helium gas (providing a low-mass-density region for Moeller electrons to escape in the forward direction), protons pass a ground plane located at a radius of $2 \mathrm{~cm}$ and then the cathode surface at a radius of $3 \mathrm{~cm}$. Upon traversing the cathode, the protons enter the sensitive ionization volume (covering radial distances from 3 to $6 \mathrm{~cm}$ ), filled with an approximately $80 \% \mathrm{He} / 20 \%$ dimethyl ether (DME) mixture. Helium as the main component of the mixture provides the necessary low density, which minimizes the energy loss of slow protons. When traversing the sensitive volume, the spectator ionizes the gas and the released electrons drift towards the amplification and readout stages (see below). The drift region voltage of the RTPC was kept at $1500 \mathrm{~V}$ for all runs. The resulting electric field produces a sufficiently short clearing time in the drift region without making the cathode voltage so high that a breakdown could occur.

The BONuS RTPC uses gaseous electron multipliers (GEMs) [51] to amplify the signal from the drift electrons. GEM foils are mechanically flexible, robust, and relatively low-cost structures, which can be used in a variety of gases and can be placed very close to readout pads, thus decreasing the effects of charge diffusion. An additional advantage is that they can be formed into nonplanar shapes; the BONuS RTPC was the first detector to use cylindrically curved GEM foils. A total of three GEM layers yielded an overall amplification factor of over 1000 during the experimental run. The GEM gain was limited by the requirement that nonlinearities (saturation) for slow spectator protons had to be avoided. This made the RTPC fairly insensitive to minimum-ionizing particles (i.e., electrons). The first GEM layer is at $6 \mathrm{~cm}$ radius, followed by two more GEM layers at 6.3 and $6.6 \mathrm{~cm}$ radius and the readout pad board at $6.9 \mathrm{~cm}$ radius. The space outside the pad board, within the bore of the solenoidal magnet, was reserved for preamplifiers and cables. The front and rear caps of the drift region are made of printed-circuit boards patterned with metal traces forming the field cage necessary to make the drift field between the concentric cylinders as close as possible to that between two infinite concentric cylinders. The overall length of the active volume is about $20 \mathrm{~cm}$.

The RTPC is segmented into two semicircular halves, each covering an azimuthal angle of around $150^{\circ}$. The readout pads have dimensions of $0.5 \times 0.45 \mathrm{~cm}$, thus covering approximately $3.5^{\circ}$ in azimuthal angle and $0.45 \mathrm{~cm}$ along the axis of the cylinder each. Pad rows along the axis of the RTPC are shifted with respect to each other to minimize the probability of a whole track being contained in the same row of pads, thus improving the track resolution. The RTPC is capable of detecting spectator protons with momenta from 0.07 to $0.15 \mathrm{GeV} / c$. Below this range, protons are stopped too soon to leave a substantial track in the RTPC, and above that range, protons are too fast, so that the radius of curvature of their trajectories is too large to confidently reconstruct their momenta (often, they are seen as infinite-momentum particles). Figure 5 shows a reconstructed RTPC event. A candidate track curved by the solenoid field is shown. The sizes of the symbols indicate the amount of charge collected on a pad. The signal was further amplified, processed by the ALICE readout system, sent to VME crates, and then sent to readout controllers within the standard CLAS data acquisition system. This system allowed us to read out approximately $1-\mathrm{kB}$ events at a rate of about $500 \mathrm{~Hz}$.

The BONuS event readout was initiated by the standard CLAS electron trigger system selecting interactions with a high probability of having an electron track in CLAS. The data recorded for each event is composed of the time slices (in 114-ns increments) and amplitudes (10 bits) of all RTPC pad signals above threshold for a time period extending from $1.7 \mu \mathrm{s}$ before to $9.7 \mu \mathrm{s}$ after a trigger. This interval is about 1.5 times the maximum drift time in the RTPC. See Ref. [46] for a detailed discussion of the BONuS RTPC. 

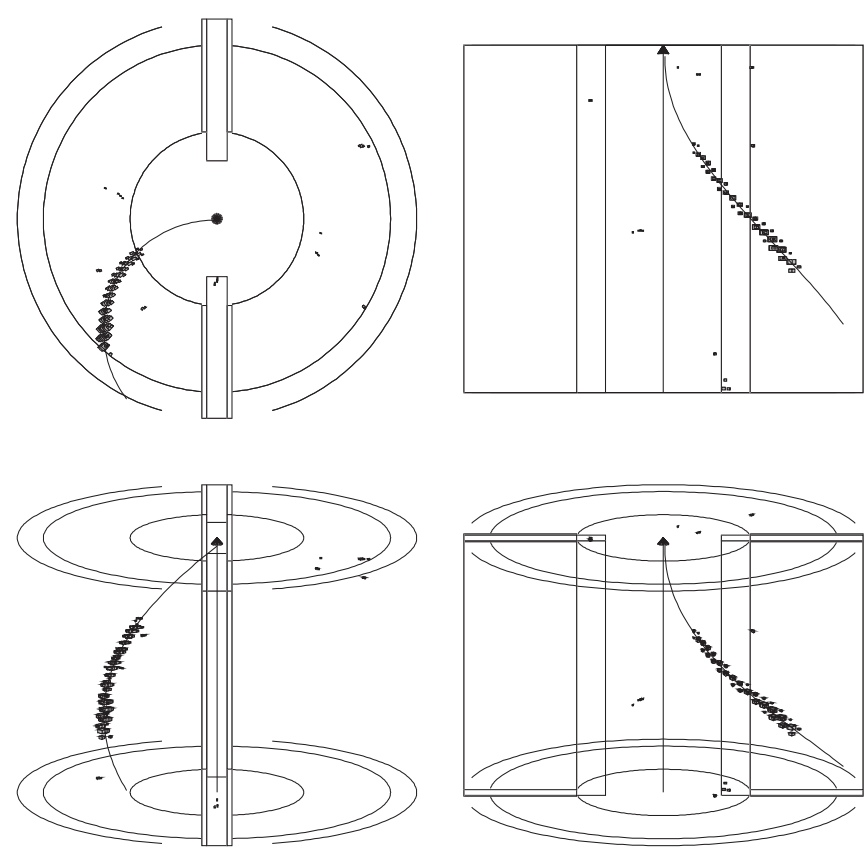

FIG. 5. An RTPC event in several views (top row, twodimensional projections on end cap and center plane; bottom row, two different three-dimensional views, the second rotated by $90^{\circ}$ ). Black blobs indicate ionized charge traced back to the spot of the ionization; solid lines going through them indicate fitted tracks. An outline of the RTPC is overlaid.

\section{ANALYSIS}

\section{A. First-pass analysis}

The analysis of the data proceeded in several steps. As a first step, all detector elements of CLAS and the RTPC were calibrated. After this, all raw digitizations written to tape were converted into reconstructed events with momentum fourvectors assigned to each identified particle. Finally, corrections to improve the tracking resolution, including effects like ionization energy loss of all charged particles, were applied. Most of these steps are part of a standard CLAS analysis (see, e.g., Ref. [52] for a more detailed description), with the exception of the work related to the RTPC, which was first used in this experiment.

\section{RTPC calibration}

Two kinds of calibrations are needed for the RTPC.

(i) Drift-velocity calibration: finding time-to-distance correspondence for drifting electrons.

(ii) Pad gain calibration: finding the correspondence between registered charge and ionization energy loss.

For the drift-velocity calibration, ionization electron paths were generated using the MAGBOLTZ program [53]. The result is a function converting any pad signal (given by the pad coordinates and the arrival time $T_{\mathrm{sig}}$ ) to a spatial point [46],

$$
(x, y, z)=f_{x y z}\left(j, T_{\text {sig }} ; V_{\text {cathode }}, V_{\mathrm{GEM}}, R_{\text {gas }}, B_{\text {sol }}\right),
$$
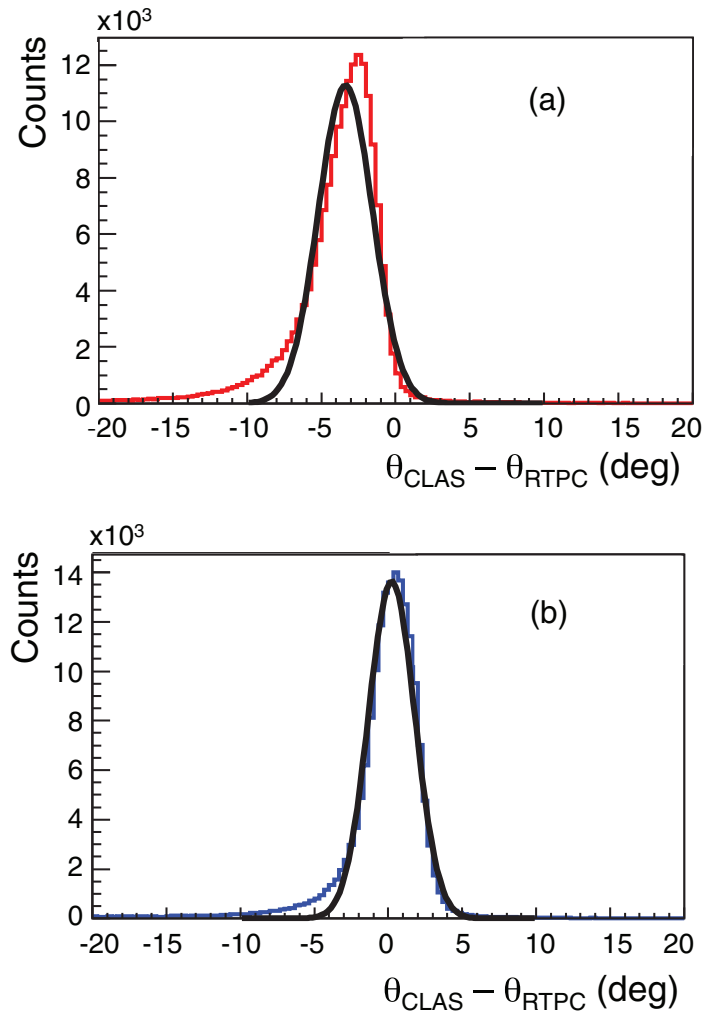

FIG. 6. (Color online) Comparison of electron-scattering angles, as reported by the RTPC and CLAS, before (a) and after (b) calibration. The comparison is shown for the left half of the RTPC; the right half results are similar. Both experimental distributions (thin colored lines) and Gaussian fits to them (thick lines) are shown.

where $j$ is the pad number and $T_{\text {sig }}$ is the time difference between the start time (given by the electron trigger) and the time when the signal was recorded at the pad. The function $f_{x y z}$ depends on the cathode voltage, $V_{\text {cathode }}$, the GEM voltage, $V_{\mathrm{GEM}}$, the solenoidal magnetic field $B_{\text {sol }}$, and the fraction $R_{\mathrm{gas}}$ of helium in the He/DME drift gas mixture.

To correct for our imperfect knowledge of the magnetic field and gas mixture as well as the start-time offset, this function was fine tuned using information from the CLAS detector. A special run with an increased RTPC voltage was conducted so that electrons registered in CLAS were also visible in the RTPC. Cross-checking information from the two detectors allowed us to find optimal parameters for the function $f_{x y z}$. Figure 6 demonstrates this comparison of track scattering angles between the RTPC and the CLAS and shows much better agreement of the angles after the final calibration of the RTPC (bottom). A similar improvement was seen in the reconstructed $z$ vertex agreement. Some minor discrepancies can still be seen in the CLAS-RTPC comparison. Those were taken care of by means of the RTPC and CLAS momentum corrections (see below).

By comparing average signal sizes from readout pads, we found that the effective detector gain varied considerably across the surface of the RTPC [46], most likely due to nonuniformities in the GEM foils or their distance from each other. Therefore, we had to accurately determine the 


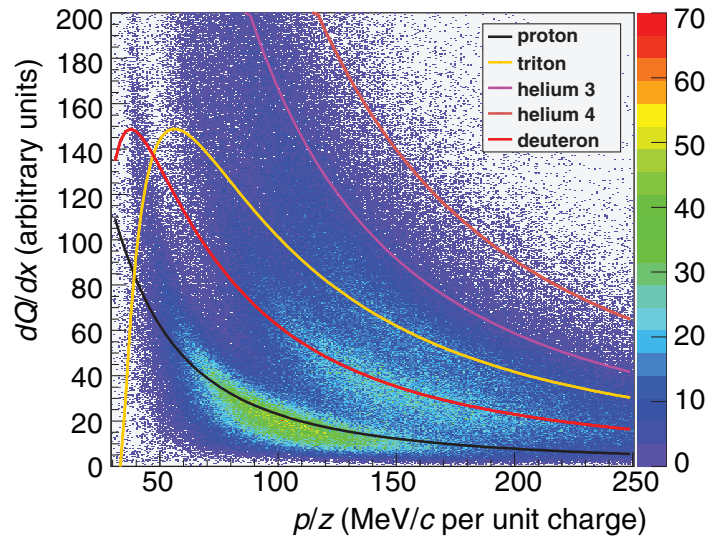

FIG. 7. (Color online) The ionization density distribution of particles registered by the RTPC after the RTPC gain calibration. The solid curves are calculated based on the Bethe-Bloch formula for $d E / d x$ for various particles, in order from bottom to top: proton, deuteron, triton, helion $\left({ }^{3} \mathrm{He}\right), \alpha\left({ }^{4} \mathrm{He}\right)$. The target was filled with ${ }^{4} \mathrm{He}$ gas and the electron energy was $2 \mathrm{GeV}$ for this measurement.

relative responses of all 3200 pads before useful $d E / d x$ information could be extracted from the data. After the driftvelocity/trajectory calibration described above, each track momentum was determined. Using the momentum, the average $d E / d x$ expected for a proton was calculated for the track using the Bethe-Bloch formula (see, for example, Ref. [54]). Using the drift paths obtained in the drift-velocity/trajectory calibration, the number of ionization electrons expected to drift to each pad $j$ was determined. Given the measured charge on that pad, we calibrated its gain $G(j)$ in an iterative procedure.

The obtained gain-normalization factors were used to scale the raw pulse heights. The same procedure was repeated excluding tracks whose measured $d E / d x$ after the first iteration was inconsistent with that of protons. The secondpass gain-normalization factors were retained and used for the final analysis. Figure 7 shows the extracted ionization density distributions after gain calibration versus measured momentum, with the expected functional correlation (from the Bethe-Bloch formula for energy loss $d E / d x$, which should be proportional to ionization per unit length) overlaid. One can clearly distinguish several bands belonging to final-state protons, deuterons, and heavier nuclei (for these data, the target was temporarily filled with ${ }^{4} \mathrm{He}$ gas).

\section{RTPC momentum corrections}

To determine spectator proton momenta at the vertex from the measured track curvature within the annulus of the sensitive drift region (ranging from 3 to $6 \mathrm{~cm}$ from the beam axis), two additional corrections were applied.

(i) The track curvature itself was corrected for possible biases in fitting a helical track to the observed ionization pattern, as well as for finite position resolution, magnetic field inhomogeneities, and possible deviation of the ideal (simulated) drift paths and drift velocities from the actual ones. (ii) The corrected curvatures were then converted to momenta at the vertex, after accounting for energy loss in the target gas and the intervening material before reaching the sensitive drift volume.

The mapping between measured curvature and vertex momentum was based on a GEANT4 simulation [55]. A large number of events was generated over the full range of target $z$ (coordinate along the beam axis) and spectator proton momenta and angles, $p_{s}, \theta, \phi$. They were subsequently run through a full simulation of the RTPC including signal conversion and track reconstruction. By comparing the results of the simulation (in terms of the reconstructed radius of curvature and angle $\theta$ of the tracks) with the thrown momenta, we extracted a one-to-one correspondence between the measured radius of curvature and the vertex spectator momentum, accounting for energy loss (see Ref. [56] for more details).

To improve the accuracy of the momentum reconstruction, we used fully exclusive ${ }^{2} \mathrm{H}\left(e, e^{\prime} p \pi^{-} p\right)$ events, where the first three particles were detected with CLAS and the last proton with the RTPC. We compared the missing momentum from the electron, pion, and proton measured in CLAS with the reconstructed momentum of the proton detected in the RTPC. The average agreement of these two quantities was optimized by adjusting the six parameters of the following correction formulas:

$$
\begin{aligned}
R_{\text {new }} & =R_{\text {old }} /\left(1+p_{1} R_{\text {old }}+p_{2}\right), \\
\theta_{\text {new }} & =\left(1+p_{3}\right) \theta_{\text {old }}+p_{4}, \\
\phi_{\text {new }} & =\left(1+p_{5}\right) \phi_{\text {old }}+p_{6},
\end{aligned}
$$

where $R_{\text {new }}$ and $R_{\text {old }}$ are the corrected and reconstructed radius of curvature, respectively, $\theta_{\text {new }}$ and $\theta_{\text {old }}$ are the corrected and reconstructed polar angle, respectively, and $\phi_{\text {new }}$ and $\phi_{\text {old }}$ are the corrected and reconstructed azimuthal angle, respectively. $p_{1}, \ldots, p_{6}$ are the fit parameters. All parameters turned out to be small, leading to corrections of order $2 \%$ on $R$ and less than $1 \mathrm{mrad}$ on $\theta$ and $\phi$.

The RTPC-measured momentum distribution of coincident protons after these two corrections was similar to the one expected from the pure spectator picture (given by the deuteron wave function in momentum space), although the measured spectrum falls off somewhat faster than predicted. This can be attributed to the RTPC reconstruction efficiency which falls off for higher spectator momenta (due to insufficient charge and track curvature for a reliable track reconstruction). We were able to partially correct this efficiency falloff using the ratio of the number of fully exclusive ${ }^{2} \mathrm{H}\left(e, e^{\prime} p \pi^{-} p\right)$ to ${ }^{2} \mathrm{H}\left(e, e^{\prime} p \pi^{-}\right) X$ events, where the first three particles in either case were detected with CLAS and we looked for the inferred proton in the RTPC.

\section{CLAS momentum corrections}

Momenta of particles reconstructed with CLAS were also corrected for minor imperfections (wire misalignments, torus and solenoid magnetic field deviations from the ideal field maps used in the reconstruction, beam offset from the ideal center line) and effects like multiple scattering and energy loss. 
These corrections have been applied and studied in previous experiments [40,57]. We determined correction parameters using a fit to fully exclusive $\mathrm{BONuS}$ data $(e p \rightarrow e p$ and $e p \rightarrow e p \pi^{+} \pi^{-}$reactions), following the method described in Ref. [40]. After applying all corrections, both the centroid and the widths of the proton missing-mass peaks were well within the established CLAS resolution and accuracy.

\section{B. Event selection and background subtraction}

\section{Particle ID cuts}

For the selection of semi-inclusive $\mathrm{D}\left(e, e^{\prime} p_{s}\right) X$ events, we developed criteria to identify scattered electrons, $e^{\prime}$, detected by CLAS, and spectator protons, $p_{s}$, detected by the RTPC.

Trigger particles were identified as electrons if they passed the following selection cuts.

(i) Track curvature consistent with a negative charge.

(ii) Cherenkov counter signal above the equivalent of two photoelectrons for momenta below $3.0 \mathrm{GeV} / c$. Above this limit, pions can emit Cherenkov radiation and the $\mathrm{CC}$ becomes inefficient for pion discrimination. (We still required a signal above the equivalent of one photoelectron in this case, to discriminate against heavier particles like kaons and protons.) In addition, geometrical and temporal matching between the $\mathrm{CC}$ signal and the measured track was required to eliminate coincidences between $\mathrm{CC}$ noise and charged-particle tracks, which can result in pions masquerading as electrons [58].

(iii) Total energy deposited in the EC above a momentumdependent threshold consistent with the EC shower sampling fraction of $\approx 0.25-0.3$.

(iv) At least $0.06 \mathrm{GeV}$ visible energy in the first (front) layers of the EC, which is significantly higher than that expected for minimum-ionizing particles like pions.

(v) Track within the fiducial volume (part of the detector with high detection efficiency and no physical obstructions).

In addition, the momentum of the trigger electron was required to be larger than $20 \%$ of the beam energy to avoid the kinematic region where radiative corrections and backgrounds become fairly large.

Spectator protons were defined by the following selection cuts:

(i) reliable fit of the track in the RTPC $\left(\chi^{2} /\right.$ degree of freedom of the fit less than 4);

(ii) positively charged particle;

(iv) more than five pads register above-threshold charge;

(v) energy loss $d E / d x$ consistent with that expected for protons (see Fig. 7; particles with energy loss more than 2 standard deviations above or less than 3 standard deviations below the measured proton $d E / d x$ distribution were rejected);

(vi) beginning and end point of the ionization trail reconstructed by the RTPC within $0.5 \mathrm{~cm}$ of the corresponding physical chamber boundary (this is basically a timing cut, because out-of-time tracks will be reconstructed at the wrong radial positions).

(vii) $z$ coordinate of the vertex is inside the fiducial target region (between $-6 \mathrm{~cm}$ and $+10 \mathrm{~cm}$ of the RTPC center).

In addition, for good electron-proton coincidence events we required that the difference between the $z$ coordinate of the electron vertex, $z_{e}$, as reconstructed by CLAS, and the $z$ coordinate of the proton vertex, $z_{p}$, as reconstructed by the RTPC, be no larger than $1.5 \mathrm{~cm}$ (to exclude accidental coincidences; see below).

Coincident events that passed all cuts were registered in four-dimensional bins in the kinematic variables $x^{*}$ or $W^{*}, Q^{2}$, $p_{s}$, and $\cos \theta_{p q}$. In addition, all electron events from inclusive $\mathrm{D}\left(e, e^{\prime}\right) X$ that pass the electron cuts above were accumulated in bins of scattered-electron energy, $E^{\prime}$, and angle, $\theta_{e}$.

\section{Accidental background subtraction}

While the cut on the distance between electron and proton vertices (see above) removes most of the accidental coincidences, the remainder (when the trigger electron and an unrelated RTPC proton happen to originate within $1.5 \mathrm{~cm}$ from each other) must be quantified and subtracted.

Such random coincidences can be simulated by taking the trigger electron from one event (without requiring a matching proton) and the RTPC proton from another event. Because spectator protons are distributed rather uniformly in angle (see Sec. V), such pairs provide very good proxies for true random coincidences. Using kinematic information from the chosen electron-proton random pair, all quantities in which real data are binned, $Q^{2}, W^{*}, x^{*}, p_{s}$, and $\cos \theta_{p q}$, are calculated, and the coincidence assigned to the corresponding bin. If the distance between the vertices of the electron and the proton, $\Delta z=z_{e}-z_{p}$, is less than $1.5 \mathrm{~cm}$, the event would emulate a random coincidence under the signal. If $\Delta z$ is larger than $2 \mathrm{~cm}$, we consider it a "wing" event. Then, after going over all the events within a bin, we form a scaling ratio, $R_{\mathrm{acc}}$, of the number of coincidences under the signal divided by the number of wing events, separately for each of our kinematic bins.

All same-event experimental coincidences between electrons and RTPC protons are separated into the same categories, wing events (those with $|\Delta z|>2 \mathrm{~cm}$ ) and "signal" (peak) events (those with $|\Delta z|<1.5 \mathrm{~cm}$ ). Then, the number of observed wing events is scaled by the ratio $R_{\text {acc }}$ to yield the number of random coincidences under the peak. The resulting accidental background events are subtracted from the events within the peak for each kinematic bin.

A sample of the distribution of both same-event and scaled random coincidences is shown in Fig. 8; the solid histogram shows the distribution of coincident events from the same "beam bucket" while the dashed line shows the simulated random distribution, normalized to the wings (outside $\pm 2 \mathrm{~cm}$ ). One can clearly see that our method leads to an excellent approximation of the accidental background in the wings. After subtracting the accidental distribution from the data, the 


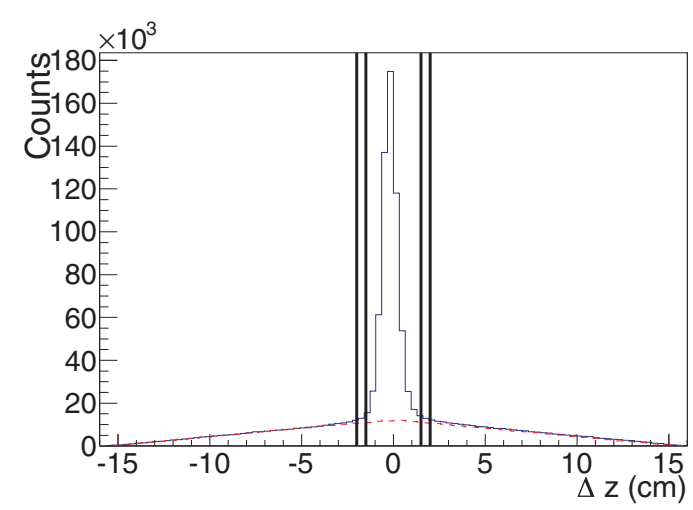

FIG. 8. (Color online) Representative plot of the $\Delta z$ distribution for coincidences (solid histogram) between electrons (in CLAS) and spectator protons (in the RTPC) from the $5-\mathrm{GeV}$ data set. Inner vertical lines indicate the region selected for data analysis $(-1.5, \ldots,+1.5 \mathrm{~cm})$. The dashed line indicates the corresponding distribution for accidental coincidences obtained by matching tracks from different events (see text), cross-normalized to the data outside the outer vertical lines $(-2.0, \ldots,+2.0 \mathrm{~cm})$. The good agreement in the "wings" outside those lines indicates that the shape of the background is well represented by this method. See text for more details.

remaining distribution is well described by a Gaussian with a resolution of about $0.7 \mathrm{~cm}(1 \sigma)$.

\section{Pair-symmetric and pion contamination}

Electron-scattering experiments typically have to account for contamination of the electron sample by $e^{+} / e^{-}$pairsymmetric contributions as well as the possible contribution from negative pions misidentified as electrons.

Pair-symmetric background comes from Dalitz decays $\left(\pi^{0} \rightarrow \gamma e^{+} e^{-}\right)$and photons converting to $e^{+} / e^{-}$pairs inside the target enclosure. The decay electron can then be misinterpreted as a scattered beam electron. The rate of this background (at most a few percent of the electron rate) has been extensively studied in previous CLAS experiments [57] for the case of inclusive electron scattering off isoscalar targets (like deuteron) and can be parametrized with a simple exponential in both electron and pion momentum and angle. This parametrization was applied as a correction to the inclusive $\mathrm{D}\left(e, e^{\prime}\right) X$ data (between $0 \%$ and $3 \%$, with an average of about $1 \%$ ). For the tagged data, the correction should be even smaller because it is proportional to the rate of $\pi^{0}$ and photon production off the neutron in deuteron (all other channels are automatically subtracted in our treatment of accidental backgrounds). We therefore did not correct the tagged data and instead included an overall systematic uncertainty of $1 \%$ due to pair-symmetric backgrounds.

Negative pions can be misidentified as electrons if they pass all cuts. The size of this contamination was studied in great detail for similar kinematics in an earlier experiment [57], and it was found to be at most $1 \%-2 \%$ for the same set of electron cuts we applied in this work. Because this correction is small compared to other possible systematic effects, it was not applied to the data but included in the total systematic uncertainty budget.

\section{Monte Carlo-based analysis}

To extract quantities of interest from the backgroundcorrected yields, we used two different analysis methods. The first one uses a full Monte Carlo simulation of the experiment to correct for acceptance effects ("Monte Carlo method"), while the second one is based on ratios of measured quantities only ("Ratio method"). The Ratio method was used for the extraction of the free neutron structure function $F_{2}^{n}$ reported by Baillie et al. [24] and in this paper; it is summarized in Sec. IV D. Some additional results reported below cover a larger range in spectator momenta and angles of the spectator proton relative to the momentum transfer vector $\mathbf{q}$ and were obtained using the Monte Carlo method, which is described in detail in the following. We show a comparison of the results obtained with both methods in Sec. V C.

\section{Event generator}

For the Monte Carlo-based analysis, we simulated both tagged $\mathrm{D}\left(e, e^{\prime} p_{s}\right) X$ events (where $p_{s}$ is the spectator proton) and fully inclusive $\mathrm{D}\left(e, e^{\prime}\right) X$ events (to determine empirical detector inefficiencies not accounted for by our simulation). For both processes, we used the same-event generator to (at least partially) cancel model dependencies. We included two basic processes in the generator.

(i) Elastic scattering off deuteron, $\mathrm{D}\left(e, e^{\prime}\right) \mathrm{D}$. We used the well-known deuteron form factors [59] and the prescription by Mo and Tsai [60] to estimate the radiative tail contribution from this process to $\mathrm{D}\left(e, e^{\prime}\right) X$, which turned out to be a very small correction to the inclusive cross section in our region of interest. (Obviously, it does not contribute at all to the tagged cross section).

(ii) Quasifree scattering off either a proton or a neutron inside deuteron, within a simple plane-wave spectator approximation. This process was further subdivided into quasielastic scattering (where the struck nucleon stays intact) and inelastic scattering off one nucleon (with the other being a spectator). These two processes are described in more detail below. Our generator did not contain additional processes like coherent pion production, FSIs, and other two-nucleon effects; therefore, the ratio of measured to simulated tagged data can be interpreted as a direct test of the spectator picture. However, these processes do not affect the overall strength of the inclusive cross section significantly except perhaps in the dip region between the quasielastic and the $\Delta$ resonance peak.

To simulate scattering off a bound nucleon inside deuteron, we used a simple spectator formalism where one nucleon is considered to be on shell and does not participate in the reaction while the other one is off the mass shell. In this picture, the energy and momentum of the off-shell bound nucleon $p^{\mu}=(E, \mathbf{p})$ are related to the spectator nucleon momentum 
$\mathbf{p}_{s}$ as

$$
\begin{aligned}
& E=M_{d}-\sqrt{M^{2}+p_{s}^{2}}, \\
& \mathbf{p}=-\mathbf{p}_{s},
\end{aligned}
$$

with $M_{d}$ the deuteron mass (see Sec. II). The off-shell mass of the struck nucleon is

$$
M^{*}=\sqrt{E^{2}-p_{s}^{2}} .
$$

The initial momentum of the struck nucleon is generated at random with weight

$$
P(\mathbf{p})=|\psi(\mathbf{p})|^{2},
$$

where $\psi(\mathbf{p})$ is the Paris deuteron wave function [61] rescaled using the light-cone formalism [62] within the approach by Frankfurt and Strikman [32].

The scattered electron kinematics are generated in the rest frame of the struck nucleon. The scattered electrons are distributed according to the radiated cross section on a nucleon at rest. The distributions are kinematically corrected for the nucleon off-shell mass. The (quasi)elastic scattering cross section is given by the Rosenbluth formula,

$$
\frac{d \sigma}{d \Omega}=\left(\frac{d \sigma}{d \Omega}\right)_{\text {Point }} \frac{1}{\epsilon}\left[\tau G_{M}^{2}\left(Q^{2}\right)+\epsilon G_{E}^{2}\left(Q^{2}\right)\right] \frac{1}{1+\tau},
$$

where $\epsilon=1 /\left[1+2(1+\tau) \tan ^{2}\left(\theta_{e} / 2\right)\right]$ is the linear polarization of the virtual photon, $G_{E}$ and $G_{M}$ are Sachs form factors, and $\tau=Q^{2} /\left(4 M^{2}\right)$. We used the parametrization of the proton form factors by Arrington [12] and the parametrization of Kubon et al. [63] for $G_{M n}$ and the Galster et al. [64] parametrization for $G_{E n}$. Higher-order QED effects and the elastic radiative tail are calculated using the full prescription of Mo and Tsai [60].

Inelastic events off protons and neutrons in deuteron are generated similarly to the quasielastic ones. The cross section is evaluated using

$$
\frac{d \sigma}{d E^{\prime} d \Omega}=\left(\frac{d \sigma}{d \Omega}\right)_{\text {Point }} \frac{2 M x F_{2}\left(x, Q^{2}\right)}{\epsilon Q^{2}} \frac{1+\epsilon R\left(x, Q^{2}\right)}{1+R\left(x, Q^{2}\right)},
$$

where

$$
R=\frac{\sigma_{L}}{\sigma_{T}}=\frac{F_{2}}{2 x F_{1}}\left(1+\frac{Q^{2}}{v^{2}}\right)-1
$$

$\sigma_{L}$ and $\sigma_{T}$ being the longitudinal and transverse virtual photoabsorption cross sections. The proton and neutron structure functions are taken from Bosted and Christy [65]. Radiative effects are simulated using the code "RCSLACPOL" [66], which is based on the prescription by Mo and Tsai. The event generator also simulates the (rather small) external radiative energy loss before scattering, due to exit and entrance windows and gas in the beam path, while external radiative and other energy losses after the scattering are included in the detector simulation (see below).

The fully inclusive sample is formed by generating quasielastic and inelastic events from both the neutron and the proton (integrated over all spectator momenta), plus the radiative elastic tail from ${ }^{2} \mathrm{H}\left(e, e^{\prime}\right)^{2} \mathrm{H}$. The simulated tagged sample contains only quasielastic and inelastic scattering events off bound neutrons, with information on the generated spectator proton being kept in addition to that on the scattered electron.

\section{Detector simulation}

The generated events are then run through a Monte Carlo simulation of the experimental setup which includes external radiation and ionization losses after the scattering. The target and RTPC parts of the setup are simulated in detail using the same GEANT4-based simulation package that was used for the RTPC momentum corrections, described in Sec. IV A 2. The standard CLAS part of the setup is simulated using the existing GEANT3-based [67] package called GSIM. After particle paths through the RTPC are simulated in GEANT4, the output information at the boundary is written to files which serve as input for the GSIM package. To simulate inefficiencies of the CLAS detector, the GSIM post processing (GPP) package is run after GSIM. It makes the GSIM output look more like real data by accounting for dead scintillators and wires and adding some Gaussian smearing to the data to match the measured detector resolution.

After the generated events are tracked through the simulated detectors, one obtains files with simulated detector responses for the generated events. Finally, these files are processed by the usual data-processing program (RECSIS), the same one used for processing experimental events. After applying the same fiducial and kinematic cuts as for the experimental data, we separately accumulate simulated data from quasielastic as well as inelastic scattering off a neutron inside deuteron. These data are binned in the same kinematic bins as the experimental tagged data.

Then all events from the elastic, quasielastic, and inelastic simulations are combined, after passing inclusive electron cuts, to simulate the inclusive electron rate. Pair-symmetric and pion contamination corrections (see Sec. IV B 3) are applied to these simulated data. Because the inclusive $\mathrm{D}\left(e, e^{\prime}\right)$ cross section is well known, the ratio of the inclusive data to the simulation can be used to extract remaining inefficiencies of the trigger and of detector elements like the $\mathrm{CC}$ and the EC that were not fully implemented in our simulation. For this purpose this ratio is calculated, for each beam energy, in bins of the final electron energy and scattering angle, $E^{\prime}$ and $\theta_{e}$. The tabulated ratio is used as a weighting factor for each simulated tagged event, depending on its electron kinematics. This factor turned out to be around 0.85 on average, with a standard deviation of 0.072 around this mean. We used this standard deviation to estimate the point-to-point systematic uncertainty of this correction as $8.5 \%$.

\section{Final data set}

The remaining steps of the Monte Carlo method require us to subtract the quasielastic radiative tail from the tagged neutron data and to normalize our results to account for any remaining RTPC inefficiency not captured by the GEANT4 simulation. So, as the next step, we normalize the simulated quasielastic events (including radiative tail) on the bound neutron to the measured quasielastic strength, integrated over the region $0.88 \mathrm{GeV} / c^{2}<W^{*}<1 \mathrm{GeV} / c^{2}$, for each bin in 


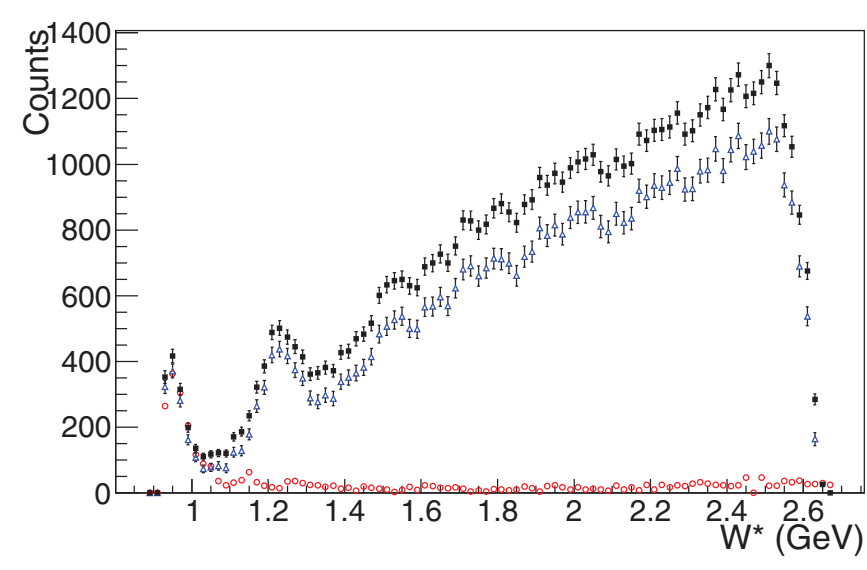

FIG. 9. (Color online) $W^{*}$ distributions (for $1.10 \mathrm{GeV}^{2} / c^{2}<$ $Q^{2}<2.23 \mathrm{GeV}^{2} / c^{2}$ ) of measured counts for the $5.3-\mathrm{GeV}$ beam energy with spectator protons detected at angles greater than about $100^{\circ}$ and momenta between 70 and $85 \mathrm{MeV} / c$. The data are shown before (upper black squares) and after subtraction of accidental coincidences and other backgrounds (lower blue triangles). Also shown are the normalized simulated counts for elastic scattering off a neutron inside the deuteron, including the radiative tail (open red circles, bottom). Note the good agreement between this simulation and the data in the quasielastic region, $W^{*}<1 \mathrm{GeV}$.

$Q^{2}$ and spectator kinematics. Figure 9 shows the resulting simulated spectrum as a function of $W^{*}$ for a specific bin in $Q^{2}$, spectator kinematics, and beam energy, together with the data before and after subtracting experimental backgrounds. The shapes of the simulated and measured spectra agree well in the region $W^{*}<1 \mathrm{GeV} / c^{2}$, giving us confidence that the radiative tail is reasonably well represented by this procedure. We then subtract this normalized simulated spectrum from the measured one over the whole $W^{*}$ range to remove the (quasi-) elastic radiative tail from the measured spectrum.

The remaining experimental spectrum is due only to inelastic ${ }^{2} \mathrm{H}\left(e, e^{\prime} p_{s}\right) X$ events and can be compared to the simulated inelastic spectrum. However, the latter must still be normalized to account for the overall efficiency of the RTPC. In particular, we find that the simulation of the RTPC response did not fully capture the experimentally observed RTPC track reconstruction efficiency within cuts, and that this efficiency varies as a function of proton momentum (from about 0.6 at the lowest $p_{s}$ down to 0.23 at the upper limit of our $p_{s}$ range). For this reason, we derive a normalization factor $N\left(p_{s}, E_{b}\right)$ for each of our four bins in spectator momentum $p_{s}$. This factor is also allowed to vary between the different time spans corresponding to each of the beam-energy settings used in our experiment (indicated by the dependence on the variable $E_{b}$ ). We determine this factor using events in the range $-1<\cos \theta_{p q}<-0.2$ (backward kinematics). According to theoretical expectations and our own data (see next section), the spectator picture works best in this kinematic region. We match the measured spectrum to the simulated one in a kinematic region where the ratio between the two is found to be flat: $W^{*}=2.0-2.2 \mathrm{GeV} / c^{2}$ for both the 4 - and the $5-\mathrm{GeV}$ data, within the lowest fully accepted $Q^{2}$ bin for each energy.
The resulting agreement between data and simulation can be seen in Fig. 13, which shows the ratio between both. This ratio fluctuates around 1.0 by about $\pm 10 \%$ in the chosen $W$ region, which is consistent with the uncertainty $\Delta N\left(p_{s}, E_{b}\right)$ we assign to the normalization factor; see next section. Note that this factor is the same for all bins in spectator angle and in $\left(W^{*}, Q^{2}\right.$ ) for a given beam energy setting and $p_{s}$ bin, allowing us to study the dependence of the data on these variables without normalization bias.

After applying the normalization $N\left(p_{s}, E_{b}\right)$ we form the ratio $R_{D / S}$ between the background and radiative tailsubtracted tagged data (integrated over a given kinematic bin) and the normalized simulation. This ratio can then be used to study the kinematic dependence of any deviations between our data and our cross-section model; see Sec. V A. If our spectator cross-section model is valid, $R_{D / S}$ can be interpreted as the ratio between the effective structure function $F_{2}^{n \text {,eff }}\left(W^{*}, Q^{2}, p_{s}, \cos \theta_{p q}\right)$ and the model input for $F_{2}^{n}\left(W^{*}, Q^{2}\right)$ for each bin:

$$
\begin{aligned}
R_{D / S} & =\frac{N_{2}^{\text {data, corr }}\left(e, e^{\prime} p_{s}\right) X}{N\left(p_{s}, E_{b}\right) N_{2}^{\text {simul }}\left(e, e^{\prime} p_{s}\right) X}\left(W^{*}, Q^{2}, p_{s}, \cos \theta_{p q}\right) \\
& =\left[1+\frac{\Delta N\left(p_{s}, E_{b}\right)}{N\left(p_{s}, E_{b}\right)}\right] \frac{F_{2}^{n, \text { eff }}\left(W^{*}, Q^{2}, p_{s}, \cos \theta_{p q}\right)}{F_{2}^{n, \text { model }}\left(W^{*}, Q^{2}\right)},
\end{aligned}
$$

where the first factor on the second line accounts for the possible normalization uncertainty.

As a further result, the value of the effective structure function $F_{2}^{n \text {,eff }}$ for a given kinematic bin in $p_{s}, \cos \theta_{q p_{s}}, Q^{2}$, and $x^{*}$ or $W^{*}$ can be extracted from the data by multiplying the ratio $R_{D / S}$ with the model input for the free $F_{2}^{n}$ at the center of that bin (thus also taking bin centering into account). This method leads to an (approximate) cancellation of the model input for $F_{2}^{n}$ because the simulated data are (roughly) proportional to it, leading to largely unbiased results for $F_{2}^{n \text {,eff }}$.

\section{Systematic uncertainties}

The total systematic uncertainty on each data point consists of an overall scale uncertainty and point-to-point uncertainties due to the various inputs and assumptions for the analysis. The scale uncertainty, $\Delta N\left(p_{s}, E_{b}\right)$, is due to our RTPC normalization method (Sec. IV C 3), which relies on the assumption that our model describes the data accurately for the kinematic bin chosen to normalize the simulated to the measured tagged inelastic data. We estimate this uncertainty by varying the $W^{*}$ range over which we compare data and simulation, which yields a scale uncertainty of $\Delta N\left(p_{s}, E_{b}\right)=$ $\pm 0.1 N\left(p_{s}, E_{b}\right)$. This includes an uncertainty of $5 \%$ for the model value for $F_{2}^{n}$ in the chosen kinematic range. This scale uncertainty is not shown on plots, because it affects all the bins in a given distribution uniformly. The remaining point-to-point systematic uncertainties are discussed below and summarized in Table I.

(i) Accidental background subtraction. Our background subtraction method (see Sec. IV B 2) depends somewhat on the limits chosen for the wings in the $\Delta z$ distribution that are used to estimate the number 
TABLE I. Point-to-point systematic uncertainties on the extracted structure function $F_{2}^{n, \text { eff }}\left(W^{*}, Q^{2}, p_{s}, \cos \theta_{p q}\right)$ from the Monte Carlo method. Each uncertainty is shown as a percentage of the structure function.

\begin{tabular}{lcl}
\hline \hline Source & Systematic uncertainty $(\%)$ & \multicolumn{1}{c}{ Explanation } \\
\hline$e^{+}$ & 1.0 & Effect of pair-symmetric contamination \\
$\pi^{-}$ & 1.0 & Effect of pion contamination \\
$\Delta z$ & 1.0 & Accidental background subtraction \\
eff $\left(E^{\prime}, \theta\right)$ & 8.5 & Uncertainty of $E^{\prime}$ - and $\theta_{e}$-dependent CLAS efficiency \\
MC & 9.0 & Combined uncertainty due to Monte Carlo statistics and systematics \\
Total & 12.5 & Added in quadrature \\
\hline \hline
\end{tabular}

of background events between the cut limits of $-1.5 \mathrm{~cm}<\Delta z<1.5 \mathrm{~cm}$. We vary the $\Delta z$ wings from the standard range $(2-16 \mathrm{~cm})$ to a smaller range of $2-9 \mathrm{~cm}$ and estimate the systematic uncertainty as the resulting change in accidental counts subtracted. This leads to an average systematic uncertainty of the order of $1 \%$ relative to the corrected data, with most bins having uncertainty under $1 \%$. Uncertainties on the subtraction of other backgrounds $\left(\pi^{-}\right.$and pair-symmetric contamination) are of the order of $1 \%$, as well.

(ii) $E^{\prime}$ - and $\theta$-dependent acceptance and efficiency uncertainty. This is the uncertainty on the estimate of the detection efficiency of the CLAS trigger electrons, calculated using the ratio of measured and simulated inclusive $\mathrm{D}\left(e, e^{\prime}\right)$ event rates (see Sec. IV C 2) as a function of $E^{\prime}$ and $\theta_{e}$. The uncertainty on this efficiency stems mostly from bin-to-bin fluctuations of the counting statistics and the uncertainty in the model used for the simulation. It was estimated by using the standard deviation of these (nearly random) fluctuations. This yields a kinematics-dependent systematic uncertainty of $8.5 \%$ (see Sec. IV C 3). (An overall scale uncertainty is already accounted for, as mentioned above).

(iii) $F_{2}^{n}$ model dependence. An overall scale uncertainty in our model of $F_{2}^{n}$ of about $5 \%$ is included in the scale factor (see above). Any remaining deviation of the model from the "true" neutron structure function is part of the information to be extracted from the ratio $R_{D / S}$ and cancels largely in the extracted values for $F_{2}^{n \text {,eff }}\left(W^{*}, Q^{2}, p_{s}, \cos \theta_{p q}\right)=R_{D / S} F_{2}^{n \text {,model }}$ because the denominator of $R_{D / S}$ is approximately proportional to $F_{2}^{n \text {,model }}$. A small residual uncertainty stems from smearing and radiative effects (that depend weakly on $F_{2}^{n \text {,model }}$ ) and the structure function $R$ used for the simulation. It is subsumed in the uncertainty due to the Monte Carlo simulation.

(iv) Monte Carlo simulations. Besides determining the detection efficiency via inclusive count rates, the Monte Carlo simulation is used in two different steps during the data analysis:

(1) to determine the quasielastic radiative tail that is subtracted from the data in the inelastic region;

(2) to calculate the ratio $R_{D / S}$ between experimental and simulated inelastic data.
Both steps entail uncertainties due to Monte Carlo statistics and possible deviations between the simulated detector response and the real performance of CLAS and the RTPC. (The separate uncertainty due to the simulation of inclusive $\mathrm{D}\left(e, e^{\prime}\right)$ rates has been discussed above).

The statistical Monte Carlo errors are calculated using simple counting statistics (Poisson distribution) and straightforward error propagation. Systematic pointto-point uncertainties are due to possible inaccuracies in our GEANT detector model and residual dependencies on the structure function models and radiative corrections [see list entry (iii)]. We kept the Monte Carlo statistical errors below the systematic uncertainties in all cases. To estimate the systematic uncertainty due to the subtraction of the radiative quasielastic tail, we compared the simulated spectra in the quasielastic region $0.9 \mathrm{GeV} / c^{2}<W^{*}<1 \mathrm{GeV} / c^{2}$ with the measured one (see, e.g., Fig. 9). We concluded that the normalization of the tail has an uncertainty of about $10 \%$, due to the slightly different shapes of these two spectra.

The systematic uncertainties due to $E^{\prime}-\theta$ efficiency, background subtraction, and Monte Carlo simulation (both parts) are added in quadrature, yielding a total point-to-point uncertainty of the ratio $R_{D / S}$ of about $12.5 \%$. To convert these values to systematic uncertainties of the $F_{2}^{n}$ structure function, they are multiplied by the value of the model $F_{2}^{n}$ in the corresponding bin. These systematic uncertainties are shown as shaded bands in all plots in Sec. V that are based on the Monte Carlo method. While they depend on kinematics, this dependence is seen to be a relatively smooth function of the kinematic variables across the various spectra shown in Sec. V.

\section{Ratio method of extracting free neutron results \\ 1. Overview of the ratio method}

The analysis method described up to this point has the advantage of using the complete available information from all detector elements of CLAS and the RTPC to correct the raw data for acceptance, efficiency, radiative effects, and backgrounds bin by bin over the full kinematic domain covered by our experiment. This is essential when studying the dependence of the extracted effective structure function $F_{2}^{n \text {,eff }}$ 
on all relevant kinematic variables. In contrast, for the purpose of extracting the (nearly) free neutron structure function $F_{2}^{n}\left(x, Q^{2}\right)$ from our data in the very important proton (VIP) region $\left(p_{s}<100 \mathrm{MeV} / c, \theta_{p q}>100^{\circ}\right)$ we used the alternative "ratio method" that is less dependent on accurate knowledge of detector efficiencies and acceptance. The first publication of BONuS results [24] is based on this approach. In this section, we give a somewhat expanded explanation of the ratio method (more details can be found in [68]). In Sec. V C we compare the results for $F_{2}^{n}\left(x, Q^{2}\right)$ from these two different analyses, which have partially independent systematic uncertainties. As can be seen from Fig. 20, the overall agreement is good and increases our confidence that all systematic experimental uncertainties of our final result have been properly accounted for.

The ratio method relies on the fact that the acceptance of the RTPC, after integration over the VIP region, is nearly independent of $W^{*}$ and $Q^{2}$ (because it depends only on the proton kinematics which are weakly correlated with these variables). Furthermore, the acceptance of CLAS for electrons within a given bin of $W^{*}$ and $Q^{2}$ for tagged events is very close to that for inclusive electrons from $\mathrm{D}\left(e, e^{\prime}\right) \mathrm{X}$ events in the equivalent $W, Q^{2}$ bin, where $W^{2}=M_{p}^{2}+2 M_{p} v-Q^{2}$ is the usual electron missing-mass variable (uncorrected for initial nucleon kinematics). We can therefore form the ratio of tagged over inclusive events, $N_{d\left(e, e^{\prime} p_{s}\right)}\left(W^{*}, Q^{2}\right) / N_{d\left(e, e^{\prime}\right)}\left(W, Q^{2}\right)$ for each bin in $W^{*}$ and $Q^{2}$ (and the same bin in $W$ ). This ratio can be related to the ratio of structure functions $F_{2}^{n}\left(W, Q^{2}\right) / F_{2}^{d}\left(W, Q^{2}\right)$ via

$$
\begin{aligned}
R_{\exp } & =\frac{N_{d\left(e, e^{\prime} p_{s}\right)}\left(W^{*}, Q^{2}\right)}{N_{d\left(e, e^{\prime}\right)}\left(W, Q^{2}\right)} C\left(E_{b}, W^{*}, W, Q^{2}\right) \\
& =\frac{F_{2}^{n}\left(W^{*}, Q^{2}\right)}{F_{2}^{d}\left(W, Q^{2}\right)} \int_{\mathrm{VIP}} d \alpha_{s} d p_{s}^{\perp} A_{p}\left(\alpha_{s}, p_{s}^{\perp}\right) S\left(\alpha_{s}, p_{s}^{\perp}\right) .
\end{aligned}
$$

Here $C\left(E_{b}, W^{*}, W, Q^{2}\right)$ is a correction factor (close to 1 ) that accounts for the slightly different acceptance (due to slightly different ranges in $E^{\prime}, \theta_{e}$ ) for inclusive electrons belonging to the bin $\left(W, Q^{2}\right)$ and tagged events belonging to the bin $\left(W^{*}, Q^{2}\right)$, as well as different radiative corrections and background contributions (see below).

The integral in Eq. (16) over the spectral function $S\left(\alpha_{s}, p_{s}^{\perp}\right)$ times the acceptance-efficiency product $A_{p}\left(\alpha_{s}, p_{s}^{\perp}\right)$ for the RTPC is largely independent of kinematics, as stated before, and taken as a normalization constant for each data-taking period (corresponding to one of the beam energy settings). It was determined by matching the extracted $F_{2}^{n} / F_{2}^{d}$ to a new fit to the world data on protons and deuterons [69]; see Sec. V B. This normalization leads to an overall scale uncertainty of $5 \%-10 \%$ (mostly due to the uncertainty on the fit). $F_{2}^{n}$ can, in principle, be obtained from the ratio $F_{2}^{n} / F_{2}^{d}$ by multiplying it with the parametrization of $F_{2}^{d}$ from Ref. [69], while the ratio $F_{2}^{n} / F_{2}^{p}$ can be calculated by multiplying with $F_{2}^{d} / F_{2}^{p}$, again from that same parametrization.

\section{Analysis details}

The ratio method used the same data set as described before, with the same corrections for RTPC and CLAS momenta, and the same kinematic cuts. The treatment of accidental background events was somewhat simplified by assuming a triangular shape for their distribution as a function of the proton-electron vertex difference $\Delta z$. This assumption is a natural consequence of the convolution of two flat distributions in $z$ and is born out by the observed shape of "truly" accidental coincidences; see Fig. 8. We then extrapolate this background from the wings (outside $\pm 2 \mathrm{~cm}$ ) of the distribution in $\Delta z$ into the signal region, $|\Delta z| \leqslant 1.5 \mathrm{~cm}$. This method gives essentially the same corrections for accidental backgrounds as the one described earlier.

The correction factor $C\left(E_{b}, W^{*}, W, Q^{2}\right)$ in Eq. (16) is composed of several contributions, accounting for the (small) difference in electron acceptance for tagged and inclusive events $\left(R_{\mathrm{acc}}\right)$, pair-symmetric $\left(C_{e}^{+}\right)$, and pion contamination $\left(C_{\pi}\right)$ and differences in radiative corrections $r_{r c}$,

$$
C\left(E_{b}, W^{*}, W, Q^{2}\right)=R_{\mathrm{acc}} C_{e}^{+} C_{\pi} r_{r c} .
$$

The correction factor $R_{\text {acc }}$ is calculated by comparing the measured inclusive rate $N_{d\left(e, e^{\prime}\right)}$ to the rate predicted by the well-known cross section for inclusive scattering off deuteron, as a function of $\left(E^{\prime}, \theta_{e}\right)$, yielding an efficiency function $\epsilon\left(E^{\prime}, \theta_{e}\right)$. This function is integrated (weighted by the data) over the range of $\left(E^{\prime}, \theta_{e}\right)$ belonging to either the bin $\left(W^{*}, Q^{2}\right)$ for tagged events or the bin $\left(W, Q^{2}\right)$ for inclusive events, and the ratio yields $R_{\text {acc }}$. Note that the overall luminosity and average event reconstruction efficiency of CLAS drop out in this ratio.

Radiated and Born cross-section models, $\sigma_{r}$ and $\sigma_{\text {Born }}$, for both electron-neutron and electron-deuteron scattering were generated by the code of Bosted and Christy [65,70] in each $\left(W^{*} / W, Q^{2}\right)$ bin. Radiative effects were again treated following Mo and Tsai [60]. In our final data sample, we avoided regions where the elastic tail contribution is larger than $10 \%$. The radiative correction is the "super-ratio"

$$
r_{r c}=\frac{\sigma_{\text {Born }}^{n} / \sigma_{r}^{n}}{\sigma_{\text {Born }}^{d} / \sigma_{r}^{d}},
$$

where indices $n$ and $d$ denote the neutron and the deuteron, respectively. Again, this factor is usually very close to 1 .

Finally, the relative contaminations of tagged and inclusive events from pair-symmetric $e^{+} e^{-}$decays and misidentified pions were estimated as described in Sec. IV B 3 and the ratios $C_{e}^{+}$and $C_{\pi}$ calculated, together with their systematic uncertainties.

All statistical errors were properly propagated from the tagged and inclusive number of counts in each bin. The systematic uncertainties of each correction factor in Eq. (17) were estimated and are listed in Table II, together with systematic uncertainties due to other sources. Even after including theoretical uncertainties (first three lines in Table II), the overall point-to-point systematic uncertainty of the extracted $F_{2}^{n} / F_{2}^{d}$ (about $7.5 \%$ ) - as well as the derived value of $F_{2}^{n}$ (about $8.7 \%$ ) - is smaller than the corresponding uncertainty of the Monte Carlo method. An overall scale uncertainty due to our cross normalization to existing fits amounts to at most $10 \%$ for each beam energy. This scale uncertainty is common to both methods (because they are both normalized to an existing parametrization of $F_{2}^{n} / F_{2}^{d}$ ) and is not included in 
TABLE II. Point-to-point systematic uncertainties on the extracted structure function ratio $F_{2}^{n}\left(W, Q^{2}\right) / F_{2}^{d}\left(W, Q^{2}\right)$ and the structure function $F_{2}^{n}\left(W, Q^{2}\right)$ derived from it, with the ratio method. Each uncertainty is shown as a percentage of the value of the result. An overall normalization uncertainty of about $7 \%-10 \%$ applies uniformly to the complete data set for each beam energy.

\begin{tabular}{lcl}
\hline \hline Source & Systematic uncertainty (\%) & Explanation \\
\hline FSI & 5.0 & Effect of FSIs [22] \\
Target fragmentation & 1.0 & Effect of target fragmentation [36] \\
Off-shellness & 1.0 & Effect of nucleon off-shellness [29] \\
$C_{e}^{+}$ & 1.0 & Effect of pair-symmetric contamination \\
$C_{\pi}$ & 1.0 & Effect of pion contamination \\
$r_{r c}$ & 2.0 & Each value of Born and radiated cross sections has an uncertainty of 1\%, \\
& & leading to a 2\% overall uncertainty \\
Int & 5.0 & Possible deviation from the assumption that the integral in Eq. (16) is constant. \\
$F_{2}^{d} / F_{2}^{p}$ & 4.2 & Fits to structure functions have point-to-point uncertainties of 3\% [65,70], \\
& & leading to a 4.2\% overall uncertainty (on extracted $F_{2}^{n}$ and $F_{2}^{n} / F_{2}^{p}$ values only) \\
Total & 8.7 & Added in quadrature \\
\hline \hline
\end{tabular}

the systematic uncertainty bands shown in the figures in the next section.

\section{RESULTS}

In the following, we present the results from our analysis of the BONuS data. We use the results derived from the Monte Carlo-based analysis to study deviations from spectator model expectations, and the ratio method results for final values of the ratios $F_{2}^{n} / F_{2}^{d}$ and $F_{2}^{n} / F_{2}^{p}$ as well as the neutron structure function $F_{2}^{n}$ in the region where the spectator model is expected to work well.

\section{A. Comparison with spectator model predictions}

The goal of this section is to assess in which kinematic region the proton spectator model describes the BONuS data and to gain a quantitative understanding of the deviations from this spectator picture. To this end, we study the dependence of the ratio of data to simulation on the kinematics of the spectator proton for different regions in $W^{*}$ and $Q^{2}$. Any systematic dependence on spectator kinematic variables would indicate deviations from the spectator model, arising, for instance, from nuclear binding modifications of the effective structure function $F_{2}^{n \text {,eff }}$, deviations from the input spectral function $S\left(\alpha_{s}, p_{s}^{\perp}\right)$, and effects from FSIs.

As outlined in the previous section, the Monte Carlobased analysis leads to extracted values for the ratio $R_{D / S}$ [Eq. (15)] and the effective neutron structure function $F_{2}^{n, \text { eff }}\left(x^{*}, Q^{2}, p_{s}, \cos \theta_{p q}\right)$ for a grid of values in $\left(x^{*}, Q^{2}\right)$ or $\left(W^{*}, Q^{2}\right)$ and averaged over bins in $\left(p_{s}, \cos \theta_{p q}\right)$. We used five bins in $Q^{2}$ with central values $0.34,0.61,0.93,1.66$ and $3.38 \mathrm{GeV}^{2} / c^{2}$ and four bins in spectator momentum: 70-85, 85-100, 100-120, and 120-150 MeV/c. The dependence of $R_{D / S}$ on the angle between the spectator momentum and the direction of momentum transfer is averaged over ten evenly spaced fine bins over the range $-1.0 \leqslant \cos \theta_{p q} \leqslant 1.0$ or, for studies of the $W^{*}$ or $x^{*}$ dependence, in three coarser bins: backward $\left(-1.0 \leqslant \cos \theta_{p q} \leqslant-0.2\right)$, sideways $(-0.2 \leqslant$ $\left.\cos \theta_{p q} \leqslant 0.2\right)$, and forward $\left(0.2 \leqslant \cos \theta_{p q} \leqslant-1.0\right)$. Similarly, $W^{*}$ is either binned finely in 90 bins of $0.03 \mathrm{GeV} / c^{2}$ width or more coarsely in six broad regions covering the quasielastic peak $\left(0.88-1.0 \mathrm{GeV} / c^{2}\right)$, the $\Delta$ resonance region $\left(1.0-1.35 \mathrm{GeV} / c^{2}\right)$, the second resonance $\left(1.35-1.6 \mathrm{GeV} / c^{2}\right)$ and third resonance $\left(1.6-1.85 \mathrm{GeV} / c^{2}\right)$ regions, and two higher- $W$ regions $\left(1.85-2.2 \mathrm{GeV} / c^{2}\right.$ and $2.2-2.68 \mathrm{GeV} / c^{2}$ ).

\section{1. $\theta_{p q}$ dependence}

The dependence of the data-to-simulation ratio on the cosine of the angle $\theta_{p q}$ gives us the most direct information on the validity of the spectator picture in different kinematic domains. In the spectator model this ratio is expected to be constant (equal to 1, modulo an overall normalization factor). Any overall trend, such as a monotonic increase or decrease with $\cos \theta_{p q}$, would indicate a shortcoming of the deuteron wave-function model, while FSI effects are expected to give rise to more complicated structures in this ratio (see Sec. II). Our data on the $\cos \theta_{p q}$ spectrum for six bins in $W^{*}$, five bins in $Q^{2}$, and four bins in $p_{s}$ are included in the supplemental material for this publication [71]. Here, we discuss a few representative plots (Figs. 10-12) of this spectrum, for $Q^{2}$ between 1.10 and $2.23 \mathrm{GeV}^{2} / c^{2}$.

Figure 10 shows the $\cos \theta_{p q}$ spectrum for a $W^{*}$ bin covering the second resonance region and four $p_{s}$ bins from the $5.25-\mathrm{GeV}$ data set. One observes first that the data lie on average about $10 \%$ higher than unity, which could be attributed to either an overall normalization error or a greater strength of the neutron structure function in this resonance region than anticipated by our $F_{2}^{n}$ model. Beyond that, it is clear that the data for the lowest $p_{s}$ bin fluctuate very little around this average (most points are less than one standard deviation away), with the possible exception of a slight increase at very forward angles (where target remnants from the struck nucleon might conceivably contribute). The fact that the $\cos \theta_{p q}$ spectrum is flat at backward angles is a clear confirmation of the spectator picture for the VIP region selected to extract the free neutron structure function. A slightly more pronounced $\cos \theta_{p q}$ dependence is seen in the next $p_{s}$ bin, and this structure becomes even more prominent for the highest two $p_{s}$ bins. This indicates that the spectator mechanism may not be as "pure" at increasing spectator momentum, as is indeed expected from FSI models. 

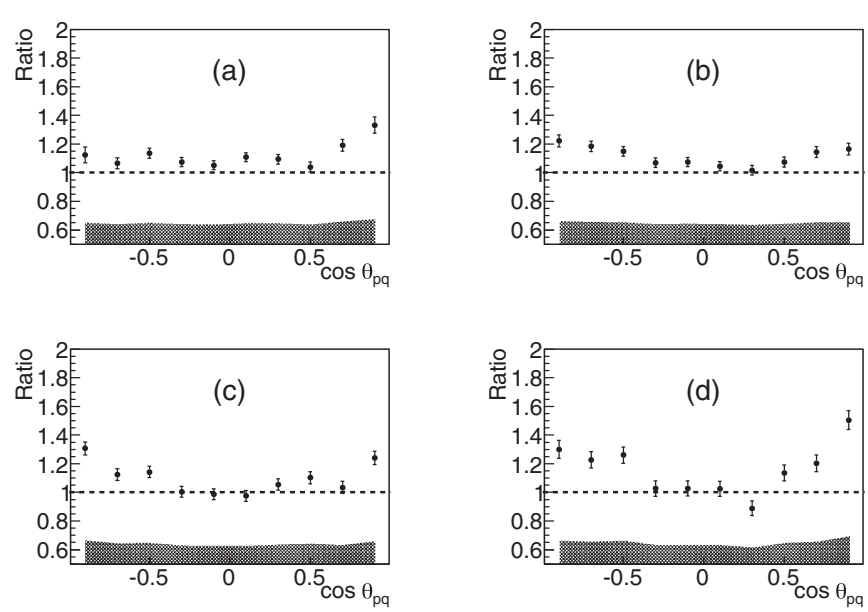

FIG. 10. Ratio $R_{D / S}$ of experimental data (with subtracted background and elastic tail) to the simulation as a function of $\cos \theta_{p q}$ for a selected bin in $Q^{2}$ (from 1.10 to $2.23 \mathrm{GeV}^{2} / c^{2}$ ) and $W^{*}$ (from 1.35 to $1.6 \mathrm{GeV} / c^{2}$ ), for a beam energy of $5.25 \mathrm{GeV}$. Each panel corresponds to one of our four bins in the spectator momentum $p_{s}$ : (a) $70-85 \mathrm{MeV} / c$, (b) $85-100 \mathrm{MeV} / c$, (c) $100-120 \mathrm{MeV} / c$, (d) $120-150 \mathrm{MeV} / c$. The error bars are statistical only, with systematic uncertainties shown as a band at the bottom.

These features become even more evident for the higher $W^{*}$ bin (at the edge of the DIS region) shown in Fig. 11. Here, the overall normalization yields an average ratio close to 1 , due to the fact that we used part of this kinematic region for our cross normalization. The structure that develops as $p_{s}$ increases shows a clear trend that is statistically significant, due to the much higher count rate in this bin. While the ratio is still mostly flat (at least within the systematic uncertainty) for backward angles and the lower two momentum bins,
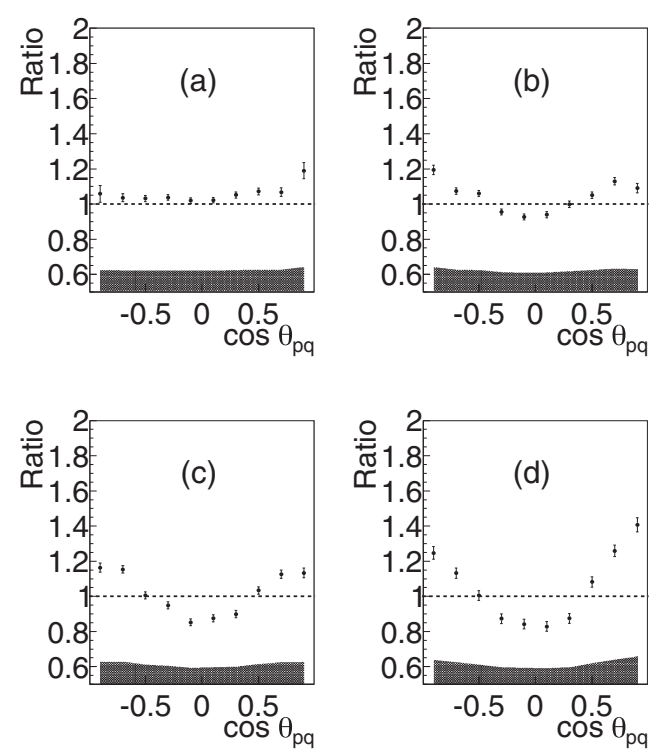

FIG. 11. The same ratio as in Fig. 10, but for a higher bin in $W^{*}$ (from 1.85 to $2.20 \mathrm{GeV} / c^{2}$ ).
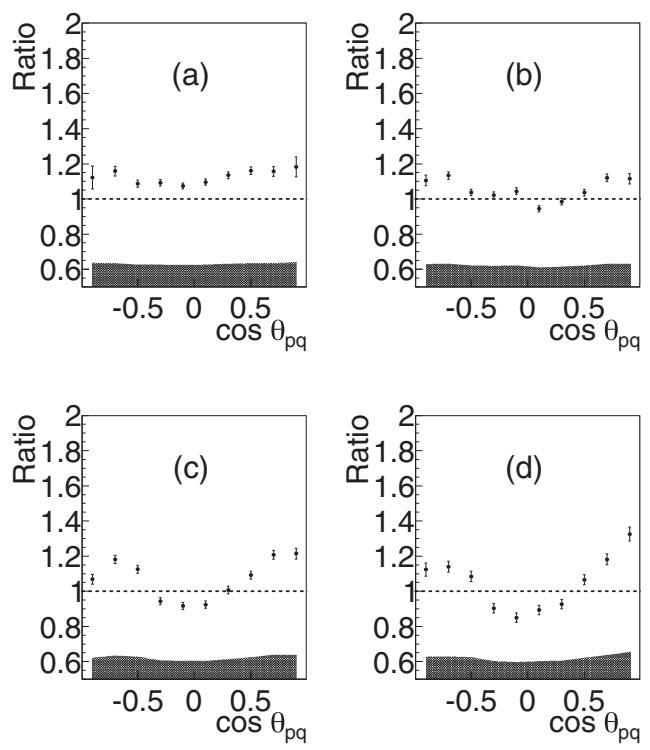

FIG. 12. The same ratio as in Fig. 11 for a beam energy of $4.23 \mathrm{GeV}$.

a significant depression at angles around $90^{\circ}$ develops at higher $p_{s}$. This is consistent with expectations from some FSI models [22,23], in which strength in this region is shifted to even higher momenta through reinteraction between the struck nucleon and the spectator. Comparison with Fig. 12 shows that the beam energy ( $4.23 \mathrm{GeV}$ in this case) has only a minor impact on the observed pattern.

Overall we find that the $\cos \theta_{p q}$ dependence is very close to flat in the backward-angle region for the two lowest $p_{s}$ bins (the region in which the spectator model should work well), for nearly all $Q^{2}-W^{*}$ bins. (Some structure visible in the second-lowest $p_{s}$ bin may, in fact, be "leakage" from higher spectator momenta, due to kinematic smearing.) This confirms that this kinematic region is described well by the spectator picture and therefore well-suited to extract (nearly) free neutron structure functions. However, significant deviations from this picture emerge at higher spectator momentum, consistent with contributions from FSI and perhaps target nucleon fragmentation. These data will enable tests and refinements of theoretical models that parametrize deviations from the spectator model [22,23,34], which in turn would allow us to correct our $F_{2}^{n}$ data for any residual effects of this kind.

\section{2. $W^{*}$ dependence}

To explore the deviations of the data from our model as a function of the invariant final-state mass, we show in Fig. 13 the ratio $R_{S / D}\left(W^{*}\right)$ for the same bin in $Q^{2}$ as before and the highest beam energy, selecting only events in which the spectator proton moves backwards relative to the momentum transfer $\left(\cos \theta_{p q} \leqslant-0.2\right)$. The four panels again show our four $p_{s}$ bins.

We note first that there appears to be an excess of events in the region below and around $W^{*}=1.2 \mathrm{GeV} / c^{2}$, above the model expectations. Some of this excess may be due to incomplete subtraction of the quasielastic radiative tail; our 

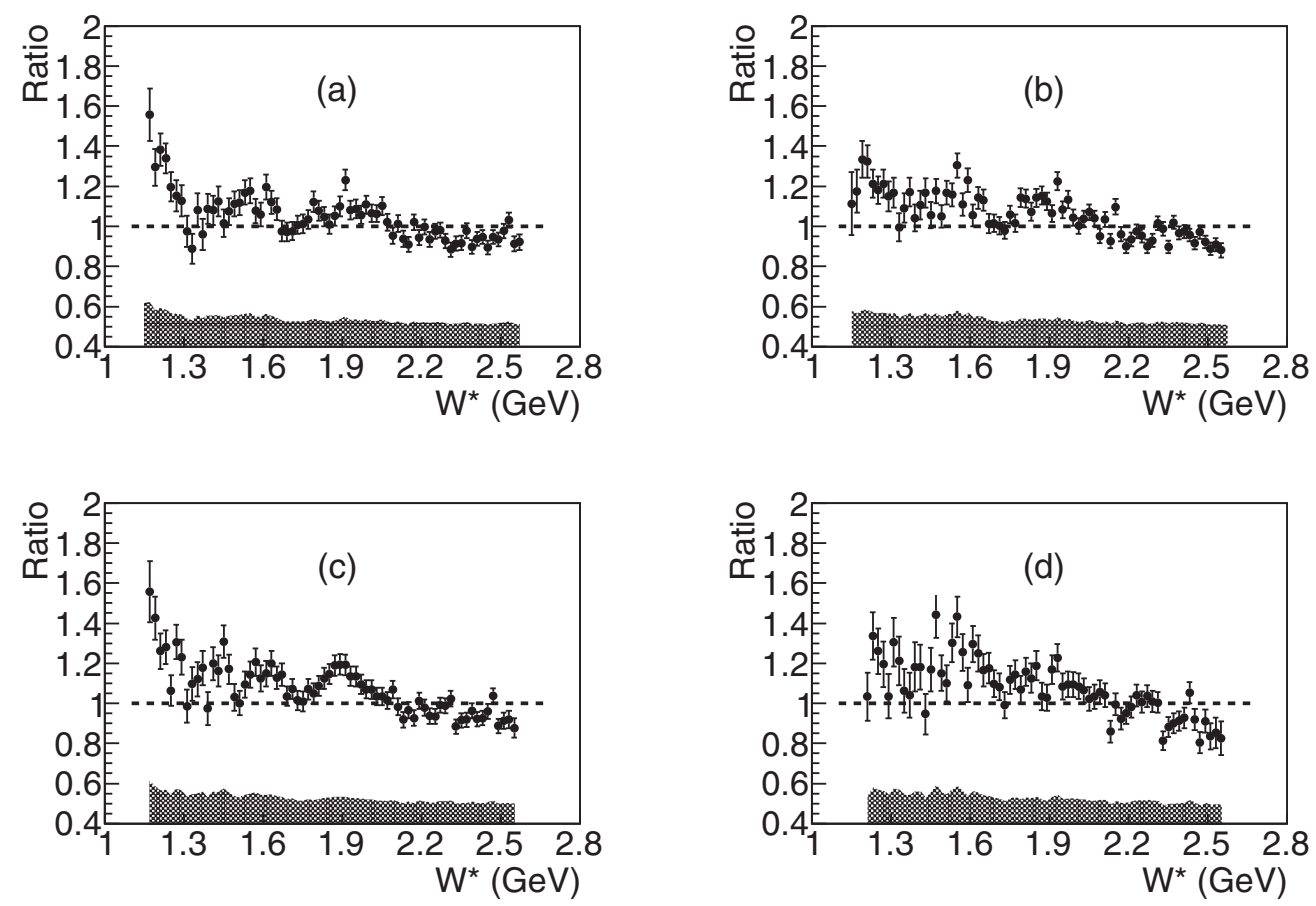

FIG. 13. Ratio of experimental data (after subtraction of accidental background and elastic tail) to the full simulation with the spectator model as a function of $W^{*}$. The data are for $Q^{2}$ from 1.10 to $2.23 \mathrm{GeV}^{2} / c^{2}$ and $\cos \theta_{p q}$ from -1.0 to -0.2 . Again, they are shown in four bins in the spectator momentum $p_{s}$ : (a) $70-85 \mathrm{MeV} / c$, (b) $85-100 \mathrm{MeV} / c$, (c) $100-120 \mathrm{MeV} / c$, (d) $120-150 \mathrm{MeV} / c$. The beam energy is $5.3 \mathrm{GeV}$. Error bars are statistical only, with systematic uncertainties shown as bands.

systematic uncertainty (shaded band) covers nearly half of the statistically significant difference. However, it is possible that our model (which is based on inclusive deuteron data) is indeed too low in this region, where $F_{2}^{n}$ varies rapidly and therefore Fermi smearing plays an important role. Similar, if somewhat smaller, enhancements are also visible in the second resonance region $\left(\right.$ around $W^{*}=1.5$ and $1.6 \mathrm{GeV} / c^{2}$ ) and between $W^{*}=$ 1.8 and $2.0 \mathrm{GeV} / c^{2}$. Because these features appear in most $p_{s}$ bins, it is unlikely that they are due to a breakdown of the spectator picture. A more recent fit to the world inclusive structure function data [69] shows better agreement with our data (see Sec. V B).

At $W^{*}>2 \mathrm{GeV}$, the data (which have been normalized to the model in the region $2.0 \leqslant W^{*} \leqslant 2.2 \mathrm{GeV}$ ) rarely differ more from our model than the combined statistical and systematic uncertainty, although one might discern a downward-sloping trend with the higher $p_{s}$ bins. Looking at the same spectra for sideways-moving spectators (see Fig. 14), we note a more pronounced depletion relative to the model at $W^{*}>2 \mathrm{GeV}$, especially for the higher $p_{s}$ bins. This could be an indication that strength in the region of higher $W^{*}$ is predominantly shifted to other kinematics (e.g., higher proton recoil momenta), due to FSI between the hadronic debris from the primary reaction and the spectator proton. Again, this is consistent with some of the existing models for FSI [22,23].

Overall, our results exhibit a generally good agreement of $F_{2}^{n \text {,eff }}\left(W^{*}\right)$ with the model for all but the lowest $W^{*}$ within the VIP (spectator) region of low $p_{s}$ bin and backward $\theta_{p q}$. Any observed structures in this region are more likely compatible with deficiencies in our $F_{2}^{n}$ model and the Monte Carlo simulation of the experiment than with a breakdown of the spectator picture.

\section{Binding effects}

We can sharpen the search for possible indications of binding and off-shell effects in our data by comparing the $x^{*}$ dependence of the effective neutron structure function for different spectator momenta. Several models of the EMC effect (see Sec. II C) suggest that the effect can be (partially) explained by a reduction of $F_{2}^{n}$ if the struck nucleon is far from its on-shell energy $E=\sqrt{M^{2}+p^{2}}$, which is equivalent within the spectator picture to a high-momentum backward-moving spectator.

We therefore plot ratios of our extracted structure functions $F_{2}^{n, \text { eff }}$ as a function of $x^{*}$ for different bins in $p_{s}$ and our usual range of backward spectator angles; see Figs. 15 and 16. The first figure is for a lower $Q^{2}$ bin, where the DIS region ends already around $x^{*}=0.35$ (indicated by arrows). It is quite apparent that the ratios are rather flat, within the statistical uncertainties, even beyond the DIS region. Systematic uncertainties largely cancel in this ratio. In particular, there is no indication of a negative slope as seen in the ratio between nuclear and nucleon structure functions (as in the EMC effect). The same behavior repeats itself for a higher $Q^{2}$ bin (Fig. 16) albeit with significantly larger statistical errors. (Here, the DIS region extends to about $x^{*}=0.52$.) While our statistical precision is not sufficient to rule out a small $p_{s}$ dependence of the structure function ratio, it appears 

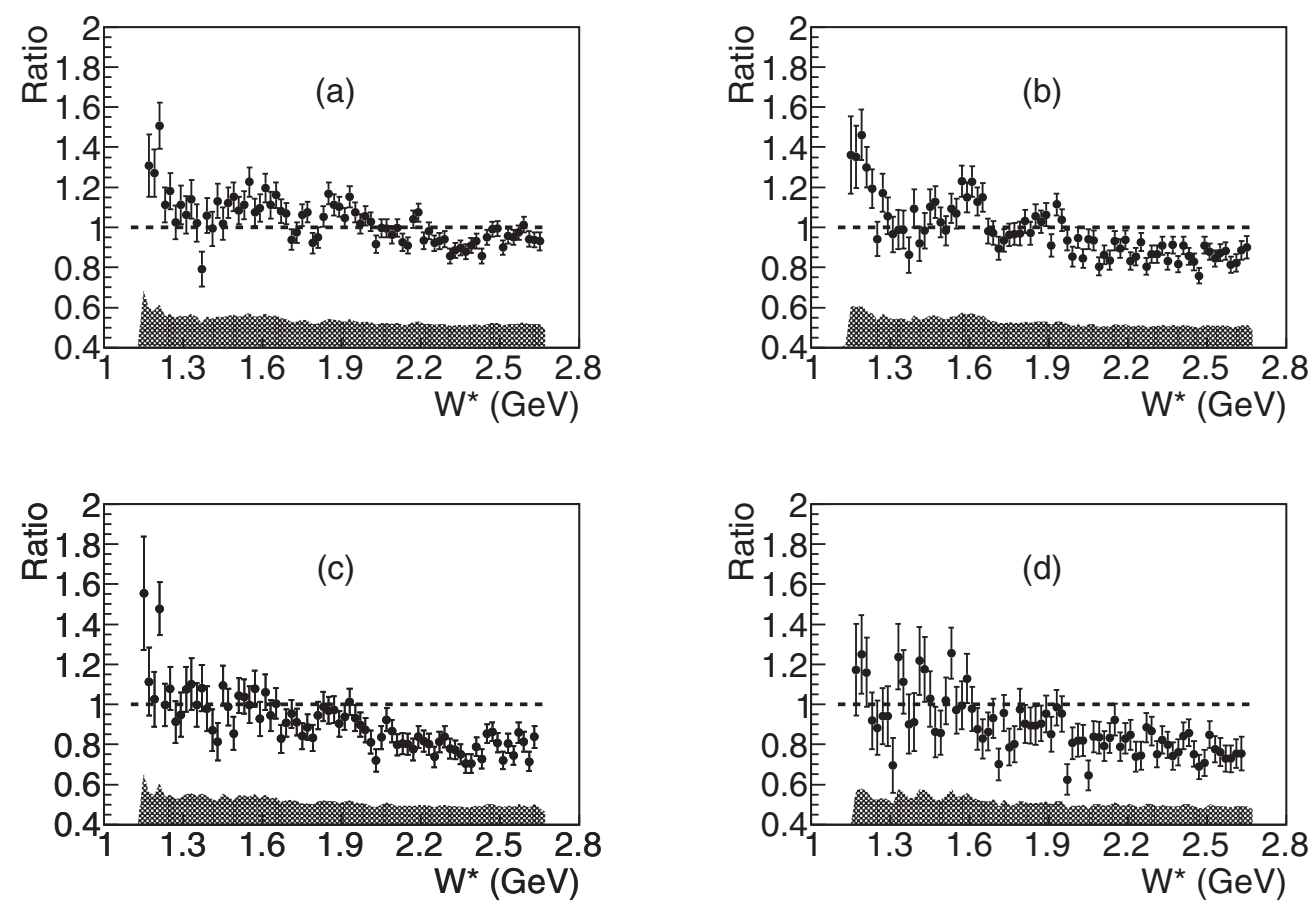

FIG. 14. Same ratio as in Fig. 13 except for sideways spectator angles, $-0.2 \leqslant \cos \theta_{p q} \leqslant 0.2$.

that binding effects are still rather small for spectator momenta up to about $150 \mathrm{MeV} / c$. The future BONuS measurement with 12-GeV beam [72] will check this conclusion with much improved precision.

\section{B. The free neutron structure function}

After establishing that the spectator picture is indeed a reasonably good approximation within our VIP region
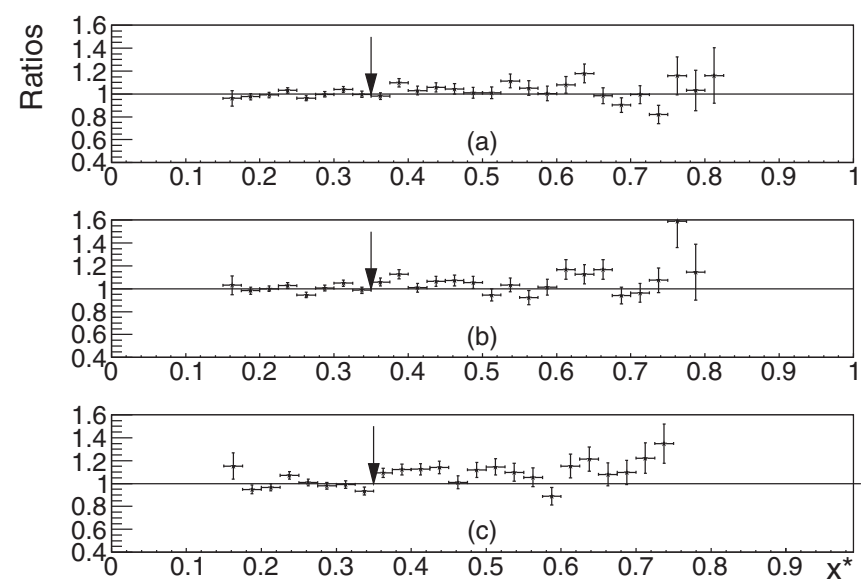

FIG. 15. Ratios of $F_{2}^{n \text {,eff }}\left(x^{*}, Q^{2}, p_{s}\right)$ for backward spectator momenta in each of the three higher $p_{s}$ bins $[(\mathrm{a})=85-100 \mathrm{MeV} / c$, (b) $=100-120 \mathrm{MeV} / c,(\mathrm{c})=120-150 \mathrm{MeV} / c]$ to $F_{2}^{n \text {,eff }}\left(x^{*}, Q^{2}, p_{s}\right)$ for the lowest $p_{s}$ bin. Data are for $Q^{2}=1.1-2.2 \mathrm{GeV}^{2} / c^{2}, \cos \theta_{p q}$ from -1.0 to -0.2 , and $5.3-\mathrm{GeV}$ beam energy. Error bars are statistical only. The arrows indicate the approximate location of the edge of the DIS region, $W^{*}=2 \mathrm{GeV}$. $\left(p_{s} \leqslant 100 \mathrm{MeV} / c, \theta_{p q} \geqslant 100^{\circ}\right)$, we proceed to extract results for the (nearly) free neutron for all kinematic bins in $W^{*}$ and $Q^{2}$, within the VIP region, using our ratio method. Because this method determines the ratio of $F_{2}^{n} / F_{2}^{d}$, we show the results for this (nearly model-independent) quantity in Figs. 17 and 18, separately for our two highest beam energies. The error bars indicate statistical errors, while the point-to-point systematic uncertainties are indicated by the band at the bottom. As explained earlier, there is an overall normalization uncertainty which means that the data must be multiplied by a factor determined from other information. For this purpose, we used a recent update of the Bosted-Christy fit [65] of the world data on protons and deuterons [69]. This new fit
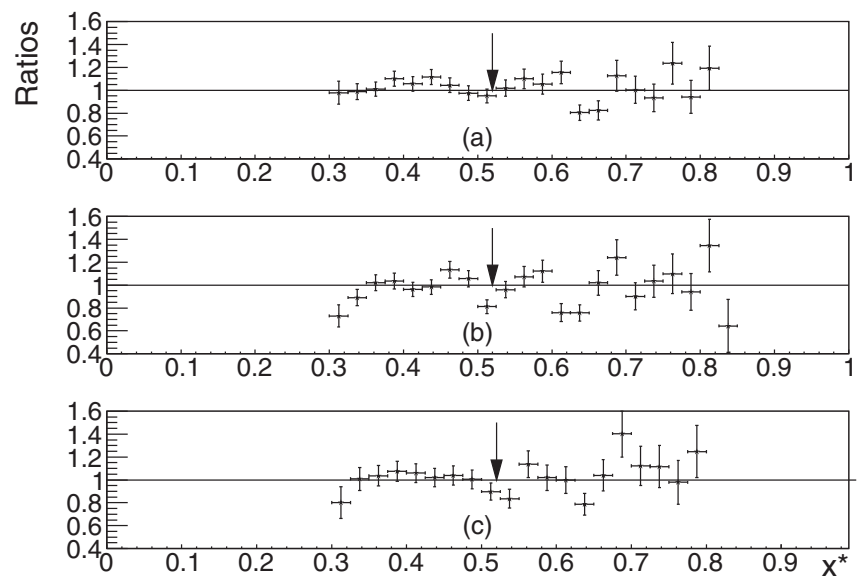

FIG. 16. Same as Fig. 15, but for a higher $Q^{2}$ bin, $2.2-4.5 \mathrm{GeV}^{2} / c^{2}$. 

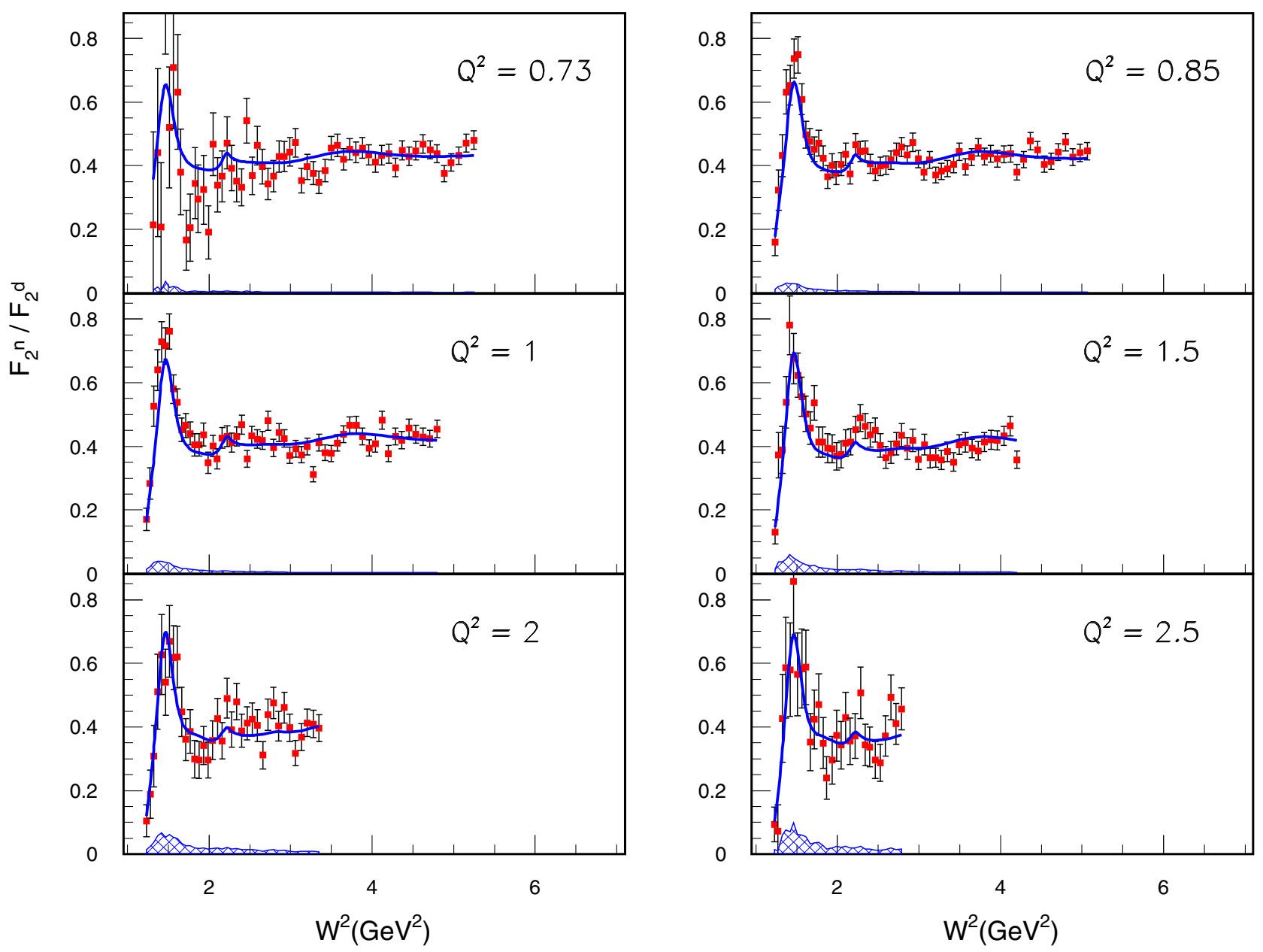

FIG. 17. (Color online) $F_{2}^{n} / F_{2}^{d}$ vs $W^{* 2}$ for $4.2-\mathrm{GeV}$ beam energy. The data are from the VIP region $p_{s} \leqslant 100 \mathrm{MeV} / c$ and $\theta_{p q} \geqslant 100^{\circ}$. Error bars indicate statistical uncertainties, while the bands show the point-to-point systematic uncertainties. The data have been normalized to the preliminary new fit of the world data by Christy et al. [69] (see text), which is shown as a solid line. We estimate that there is an overall normalization uncertainty of up to $10 \%$.

uses a convolution model [28] to combine parametrizations of proton and neutron structure functions to model the deuteron. From this fit (which does not yet include the BONuS data), the ratio $F_{2}^{n} / F_{2}^{d}$ can be extracted in a model-dependent way and we use the result to determine the overall normalization constants for both beam energies, by minimizing the $\chi^{2}$ of the normalized data versus the fit. The data shown in Figs. 17 and 18 are the main result of the BONuS experiment; they are available in tabular form in the Supplemental Material of this publication [71] and in the CLAS experimental database [73]. We estimate that the normalization uncertainty could be as large as $10 \%$, by comparing our present result to earlier fits of $F_{2}^{n} / F_{2}^{d}[65]$.

Within the assumptions of the new fit, we can also extract $F_{2}^{n}$ from the ratio by multiplying it with the fit result for $F_{2}^{d}$. Our corresponding results for $F_{2}^{n}$ as a function of $W^{* 2}$ are shown in Fig. 19 in six different slices of $Q^{2}$, with both beam energies combined. We point out that these results depend on the exact functional form used for $F_{2}^{d}$ and could change if other models are used. The underlying parametrization for $F_{2}^{n}$ from this new fit is also shown as a solid line. We note that the agreement between $F_{2}^{n}$ obtained from the two energies (after cross normalization) is quite good, increasing our confidence that smaller corrections, such as those due to radiative effects, detector acceptance, and the contribution from $R=\sigma_{L} / \sigma_{T}$ are quite small and well under control.

We also observe a generally good agreement between the data and the new fit, but with some indications for room to improve the latter. In particular, the ratio between the strength at the top of the three resonance "peaks" and the valleys in between appears larger in some of our data than in the fit. Such a deviation from the fit (which is based on inclusive deuteron world data) is understandable, keeping in mind that our experiment is the first one that does not have to rely on an unfolding prescription. The Fermi smearing for inclusive scattering off the deuteron tends to wash out strong resonance features. Ultimately, BONuS data will be incorporated into this new fit to further improve its precision in describing the neutron. 

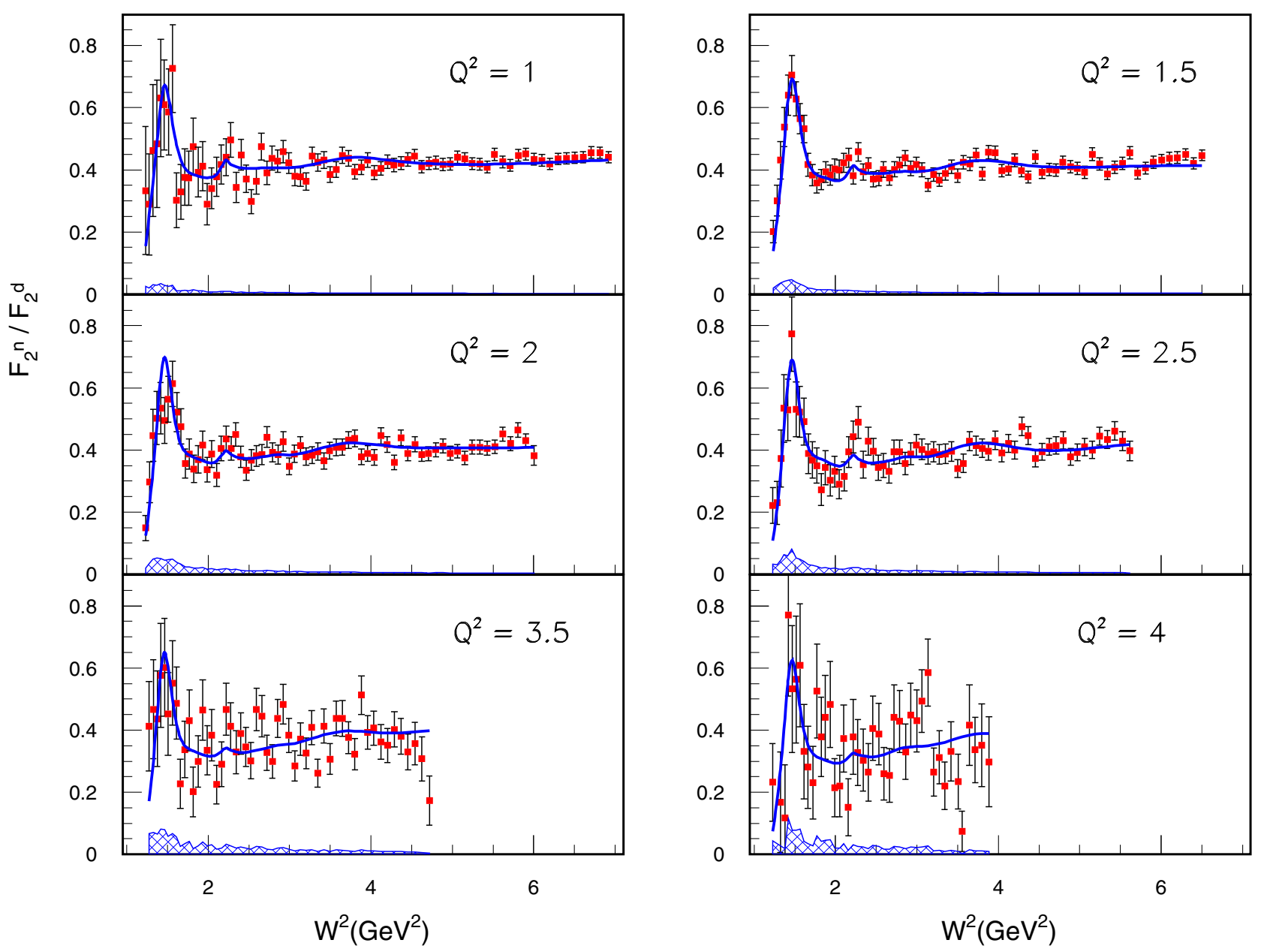

FIG. 18. (Color online) Same as Fig. 17, for 5-GeV beam energy.

\section{Results in the DIS region}

Our second main goal is to pin down the behavior of $F_{2}^{n}$ at large $x$ but in the DIS region (see Sec. II). Unfortunately, the kinematic reach of the present BONuS experiment was restricted by the maximum available beam energy $(5.25 \mathrm{GeV})$, which limits us to $x<0.55$ if we require $W^{*}>2 \mathrm{GeV} / c^{2}$. Even pushing down to $W^{*}>1.8 \mathrm{GeV} / c^{2}$ does not extend the $x$ range much beyond $x=0.6$, which is the region where presently the uncertainty on the down quark distribution function becomes large. Still, we can compare our results over the measured range $(0.2<x<0.65)$ with existing next-toleading-order fits based on world data [13]. In Fig. 20, we show our results for $F_{2}^{n}$ using both analysis methods.

For both analyses, we select events in the VIP region $\left(p_{s} \leqslant 100 \mathrm{MeV} / c\right.$, and $\theta_{p q} \gtrsim 100^{\circ}$ ) from the highest beam energy. We require $W^{*}>1.8 \mathrm{GeV} / c^{2}$ and integrate over all $Q^{2}>1 \mathrm{GeV}^{2} / c^{2}$ within a given $x$ bin. We convert the values for $F_{2}^{n} / F_{2}^{d}$ from the ratio method once again using the new fit for $F_{2}^{d}$, and for the Monte Carlo Method we multiply the ratio $R_{S / D}$ with the model for $F_{2}^{n}$ used for the generated events in our simulation. Both results are normalized at $x=0.32$ to the middle of the uncertainty band of the CJ fit [13] (given by the two solid lines in Fig. 20). In spite of significant differences between the two approaches, the results of the Monte Carlo method ("Analysis 1," inverted triangles) and the ratio method ("Analysis 2," squares) agree very well within their systematic uncertainties (given for Analysis 1 by the shaded band). We reiterate that, apart from overall normalization factors (not included in the shaded band), the systematic uncertainties of the two methods are largely uncorrelated. Most of the data are within or close to the uncertainty range of the $\mathrm{CJ}$ fit, although some fluctuations (most likely due to remaining resonant contributions) are visible. (The $\mathrm{CJ}$ band does not extend below $x=0.3$ because the fit is restricted to $Q^{2}>1.6 \mathrm{GeV}^{2} / c^{2}$ and our data fall below that value for $x \leqslant 0.3$.)

The ratio $F_{2}^{n} / F_{2}^{p}$, which is of high interest because of its relationship to the asymptotic $d / u$ ratio (see Sec. II), can also be extracted from our data using a suitable model for $F_{2}^{p}$. We showed this quantity in our previous publication [24], using the ratio method. We reproduce this result here in Fig. 21, updated with the new fit for $F_{2}^{d}$ and $F_{2}^{p}$. The results are shown for three lower cuts on the range in $W^{*}$ over which we integrate our data. The red triangles are for $W^{*}>1.8 \mathrm{GeV}$, i.e., showing the same data as in Fig. 20. They agree reasonably well with the prediction from the $\mathrm{CJ}$ fit, but do not extend much beyond $x=0.6$. The black squares $\left(W^{*}>1.6 \mathrm{GeV}\right)$ and the blue 

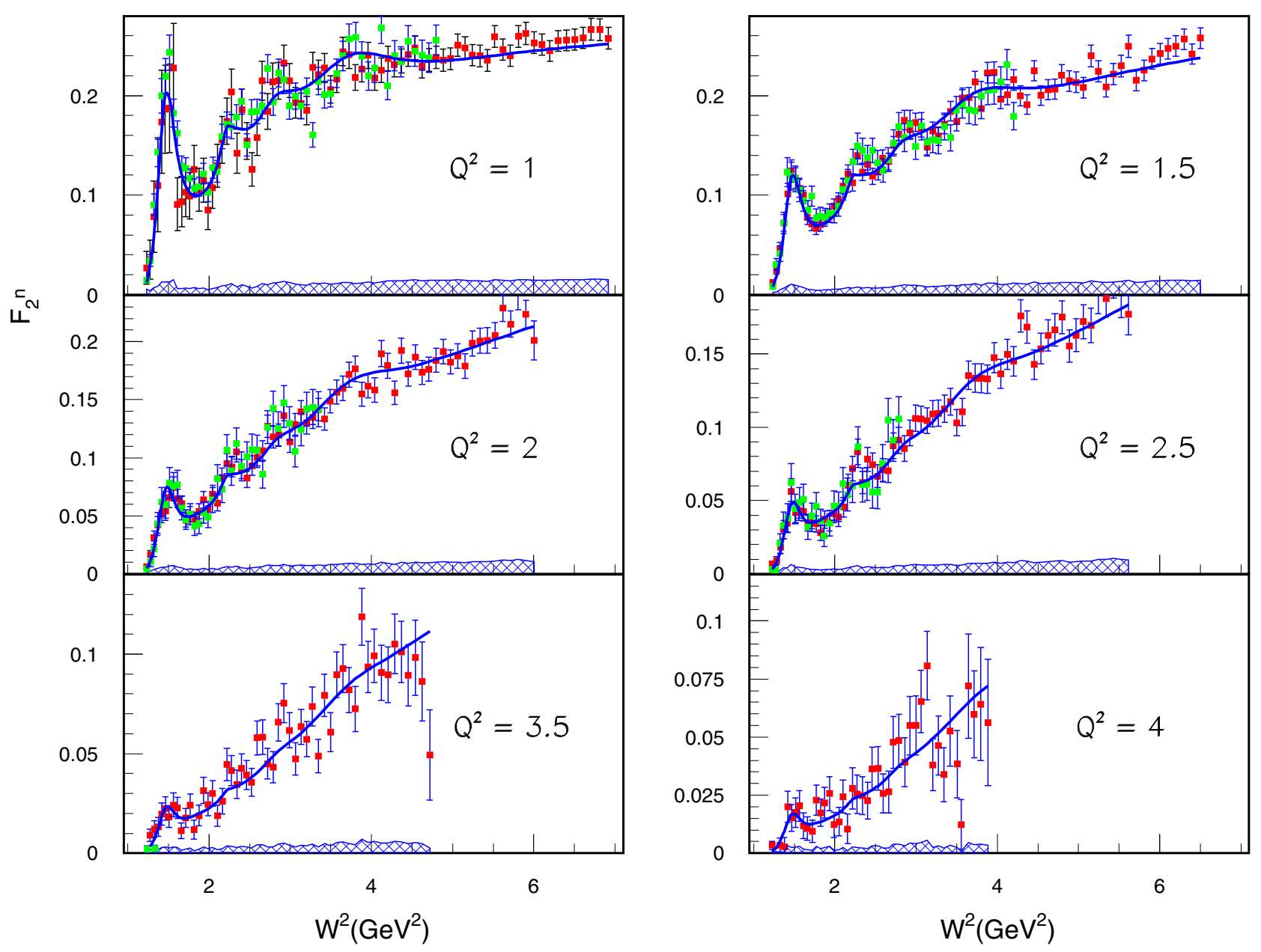

FIG. 19. (Color online) Extracted structure function $F_{2}^{n}$ as a function of squared final-state invariant mass $W^{* 2}$ for six bins in $Q^{2}$. Results are shown for two beam energies, $4.2 \mathrm{GeV}$ (green circles) and $5.3 \mathrm{GeV}$ (red squares). Error bars are statistical only, with systematic uncertainties for the 5.3-GeV data shown as a band. (Systematic uncertainties of $4.2-\mathrm{GeV}$ are very similar in magnitude to the shown ones). The solid line indicates a new fit to the world data on deuteron and proton targets that does not include BONuS data (see text).

circles $\left(W^{*}>1.4 \mathrm{GeV}\right)$ push this limit to higher $x$, but some clear resonant structure can be observed at large $x$. Taken at face value, the difference between these integration regions can be interpreted as a first hint that local duality may not hold as well for the neutron as for the proton in our kinematic region. Ultimately, only by repeating this measurement with significantly higher beam energy can one cleanly extract the DIS limit for $F_{2}^{n} / F_{2}^{p}$ as $x \rightarrow 1$. A corresponding measurement is planned for the CLAS12 spectrometer at Jefferson Lab after the upgrade to $11-\mathrm{GeV}$ beam energy is completed [72].

\section{SUMMARY}

We have presented the full analysis and final results from the BONuS experiment, which accessed for the first-time structure functions of the neutron by tagging spectator protons in the reaction ${ }^{2} \mathrm{H}\left(e, e^{\prime} p_{s}\right)$. Comparison of our data to a full Monte Carlo simulation based on the spectator model in the IA shows generally good agreement for the lowest spectator momenta $\left(p_{s}=70-85 \mathrm{MeV} / c\right)$, especially in the backward $\theta_{p q}$ region. Deviations from the spectator picture could be identified, however, at higher momenta. The results for the dependence on the spectator proton angle tend to agree with expectations from target fragmentation models $[19,36]$, with the data showing an enhancement in the region of forward $\theta_{p q}$, as well as with FSI models [22], which predict a dip in the vicinity of $\theta_{p q}=90^{\circ}$.

Within the kinematic region of its applicability, the spectator model allows us to extract the ratio $F_{2}^{n} / F_{2}^{d}$ of the free neutron structure function to the deuteron one over a wide range in $x$ or $W$ and $Q^{2}$. Comparison to a new, preliminary fit for this ratio from inclusive deuteron data using Fermismearing models [69] shows overall good agreement, but with some room for improvement in the detailed description of the resonance structures present in the data. In the DIS region, our data agree well with existing PDF parametrizations [13] out to $x \approx 0.65$, where uncertainties become large.

Structure functions extracted from the BONuS experiment using two different analysis methods are in agreement with each other, indicating that systematic uncertainties are under control. The complete data set for $F_{2}^{n} / F_{2}^{d}$ over all bins in $\left(W^{*}, Q^{2}\right)$ is available from the CLAS database [73] and as 


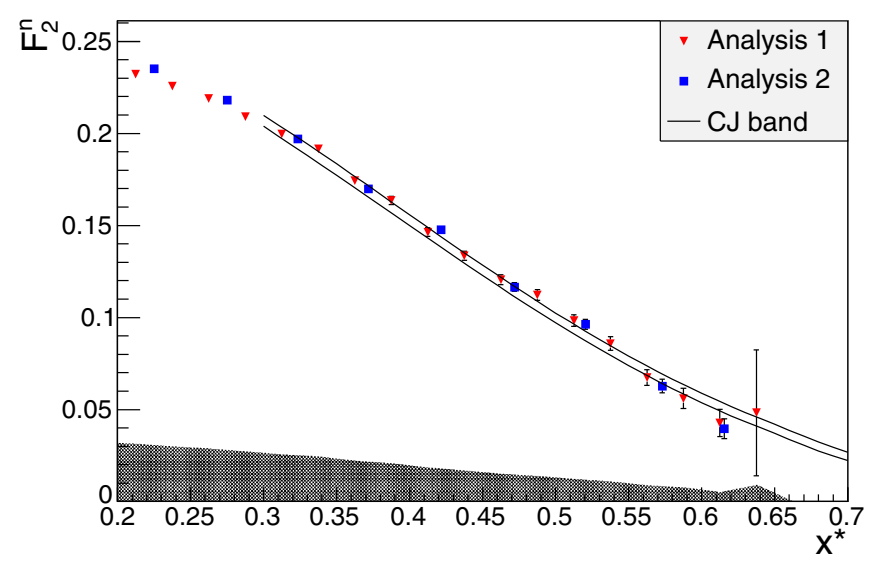

FIG. 20. (Color online) Results for the neutron structure function $F_{2}^{n}(x)$ (integrated over $Q^{2}>1 \mathrm{GeV}^{2} / c^{2}$ while requiring $W^{*}>$ $1.8 \mathrm{GeV} / c^{2}$ ) from the Monte Carlo method (labeled "Analysis 1 ") and ratio method (labeled "Analysis 2"). The range of $F_{2}^{n}$ from the CJ fit [13] is shown by the two solid lines. Systematic uncertainties for the Monte Carlo method are shown as the shaded band. The two analysis results are cross normalized to the average of the $\mathrm{CJ}$ fit at $x=0.32$.

Supplemental Material to this paper [71]. It will aid the improvement of existing models and parametrizations of neutron structure functions. These parametrizations, in turn, are crucial for other experimental goals, such as the extraction of neutron spin structure functions from polarization asymmetries, more precise studies of the nuclear EMC effect via comparisons of nuclear cross sections with the free proton and neutron cross sections, as well as reducing uncertainties in PDFs used for extracting information from collider measurements. Our data will also provide constraints on the isospin dependence of nucleon resonant excitations and the nonresonant background, as well as tests of quark-hadron duality.

A future experiment with the energy-upgraded accelerator at Jefferson Lab will allow us to both improve the statistical precision and extend the range in $x$ [72]. This experiment will finally settle the question about the asymptotic behavior of the $d / u$ ratio in the limit $x \rightarrow 1$.

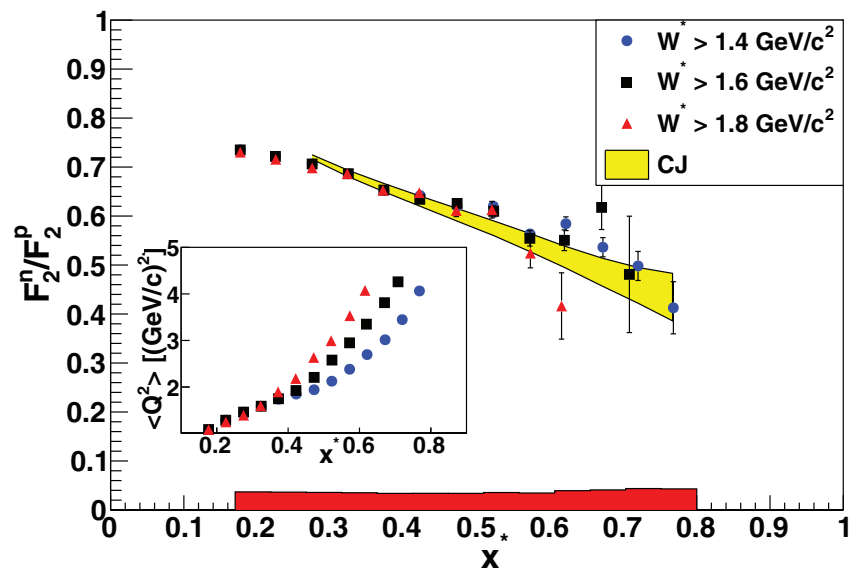

FIG. 21. (Color online) Results for the ratio of the neutron to proton structure functions $F_{2}^{n} / F_{2}^{p}(x)$ (integrated over $Q^{2}>1 \mathrm{GeV}^{2} / c^{2}$ and three different minimum values for $W^{*}$ ) from the ratio method. The uncertainty range from the CJ fit [13] is shown by the (yellow) shaded band. Systematic uncertainties are shown as the (red) shaded band at the bottom. Our data are cross normalized to the average of the CJ fit at $x=0.32$. The inset shows the average $Q^{2}$ for each data point, separately for the three lower $W^{*}$ limits.

\section{ACKNOWLEDGMENTS}

We thank the staff of the Jefferson Lab accelerator and Hall B for their support on this experiment. This work was supported in part by the Chilean Comisión Nacional de Investigación Científica y Tecnológica (CONICYT), the Italian Istituto Nazionale di Fisica Nucleare, the French Centre National de la Recherche Scientifique, the French Commissariat à l'Energie Atomique, the U.S. Department of Energy, the National Science Foundation, the Scottish Universities Physics Alliance (SUPA), the United Kingdom's Science and Technology Facilities Council, and the National Research Foundation of Korea. The Southeastern Universities Research Association (SURA) operates the Thomas Jefferson National Accelerator Facility for the United States Department of Energy under Contract No. DE-AC05-84ER40150.
[1] M. E. Christy and W. Melnitchouk, J. Phys. Conf. Ser. 299, 012004 (2011).

[2] J. Chen, A. Deur, S. Kuhn, and Z. Meziani, J. Phys. Conf. Ser. 299, 012005 (2011).

[3] W. Melnitchouk, R. Ent, and C. E. Keppel, Phys. Rep. 406, 127 (2005).

[4] R. J. Holt and C. D. Roberts, Rev. Mod. Phys. 82, 2991 (2010).

[5] P. Jimenez-Delgado, W. Melnitchouk, and J. F. Owens, J. Phys. G 40, 093102 (2013).

[6] J. Bleckwenn, J. Moritz, K. H. Schmidt, and D. Wegener, Phys. Lett. B 38, 265 (1972).

[7] M. Kobberling, J. Mortiz, K. H. Schmidt, D. Wegener, D. Zeller et al., Nucl. Phys. B 82, 201 (1974).
[8] L. M. Stuart, P. E. Bosted, L. Andivahis, A. Lung, J. Alster et al., Phys. Rev. D 58, 032003 (1998).

[9] E. D. Bloom and F. J. Gilman, Phys. Rev. Lett. 25, 1140 (1970).

[10] L. W. Whitlow et al., Phys. Lett. B 282, 475 (1992).

[11] W. Melnitchouk and A. W. Thomas, Phys. Lett. B 377, 11 (1996).

[12] J. Arrington et al., J. Phys. G 36, 025005 (2009).

[13] A. Accardi, W. Melnitchouk, J. F. Owens, M. E. Christy, C. E. Keppel, L. Zhu, and J. G. Morfín, Phys. Rev. D 84, 014008 (2011)

[14] J. Arrington, J. G. Rubin, and W. Melnitchouk, Phys. Rev. Lett. 108, 252001 (2012).

[15] J. F. Owens, A. Accardi, and W. Melnitchouk, Phys. Rev. D 87, 094012 (2013).

[16] S. Kuhlmann et al., Phys. Lett. B 476, 291 (2000). 
[17] L. T. Brady, A. Accardi, W. Melnitchouk, and J. F. Owens, J. High Energy Phys. 06 (2012) 019.

[18] L. L. Frankfurt and M. I. Strikman, Phys. Rep. 76, 215 (1981).

[19] S. Simula, Phys. Lett. B 387, 245 (1996).

[20] W. Melnitchouk, M. Sargsian, and M. I. Strikman, Z. Phys. A 359, 99 (1997).

[21] M. Sargsian and M. Strikman, Phys. Lett. B 639, 223 (2006).

[22] C. C. degli Atti, L. P. Kaptari, and B. Z. Kopeliovich, Eur. Phys. J. A 19, 145 (2004).

[23] W. Cosyn and M. Sargsian, Phys. Rev. C 84, 014601 (2011).

[24] N. Baillie et al., Phys. Rev. Lett. 108, 142001 (2012).

[25] I. Niculescu et al., Phys. Rev. Lett. 85, 1186 (2000).

[26] J. Arrington, R. Ent, C. E. Keppel, J. Mammei, and I. Niculescu, Phys. Rev. C 73, 035205 (2006).

[27] S. P. Malace, Y. Kahn, W. Melnitchouk, and C. E. Keppel, Phys. Rev. Lett. 104, 102001 (2010).

[28] Y. Kahn, W. Melnitchouk, and S. A. Kulagin, Phys. Rev. C 79, 035205 (2009).

[29] W. Melnitchouk, A. W. Schreiber, and A. W. Thomas, Phys. Lett. B 335, 11 (1994).

[30] S. A. Kulagin and R. Petti, Nucl. Phys. A 765, 126 (2006).

[31] G. B. West, Phys. Lett. B 37, 509 (1971).

[32] L. L. Frankfurt and M. I. Strikman, Phys. Rep. 160, 235 (1988).

[33] L. P. Kaptari and A. Y. Umnikov, Phys. Lett. B 259, 155 (1991).

[34] L. L. Frankfurt, W. R. Greenberg, G. A. Miller, M. M. Sargsian, and M. I. Strikman, Z. Phys. A 352, 97 (1995).

[35] M. R. Adams et al., Phys. Rev. Lett. 74, 5198 (1995).

[36] C. C. degli Atti and S. Simula, Phys. Lett. B 319, 23 (1993).

[37] G. D. Bosveld, A. E. L. Dieperink, and A. G. Tenner, Phys. Rev. C 49, 2379 (1994).

[38] L. Heller and A. W. Thomas, Phys. Rev. C 41, 2756 (1990).

[39] F. Gross and S. Liuti, Phys. Rev. C 45, 1374 (1992).

[40] A. V. Klimenko et al., Phys. Rev. C 73, 035212 (2006).

[41] M. D. Mestayer et al., Nucl. Instrum. Methods Phys. Res., Sect. A 449, 81 (2000).

[42] G. Adams et al., Nucl. Instrum. Methods Phys. Res., Sect. A 465, 414 (2001).

[43] E. S. Smith et al., Nucl. Instrum. Methods Phys. Res., Sect. A 432, 265 (1999).

[44] M. Amarian et al., Nucl. Instrum. Methods Phys. Res., Sect. A 460, 239 (2001).

[45] B. A. Mecking et al., Nucl. Instrum. Methods Phys. Res., Sect. A 503, 513 (2003).

[46] H. Fenker et al., Nucl. Instrum. Methods Phys. Res., Sect. A 592, 273 (2008).

[47] K. H. Ackermann, F. Bieser, F. P. Brady, D. A. Cebra, J. E. Draper et al., Nucl. Instrum. Meth. A 499, 713 (2003).
[48] G. Agakishiev et al., Nucl. Phys. A 661, 673 (1999).

[49] R. E. Bosch, A. J. de Parga, B. Mota, and L. Musa, IEEE Trans. Nucl. Sci. 50, 2460 (2003).

[50] L. Musa, Nucl. Phys. A 715, 843 (2003).

[51] F. Sauli, Nucl. Instrum. Methods Phys. Res., Sect. A 386, 531 (1997).

[52] S. Tkachenko, Ph.D. thesis, Old Dominion University, 2009, http://www.jlab.org/Hall-B/general/thesis/Tkachenko_thesis. pdf

[53] S. Biagi, Nucl. Instrum. Methods Phys. Res., Sect. A 421, 234 (1999), The MAGBOLTZ programme, version 2 (2005).

[54] W. Leo, Techniques for Nuclear and Particle Physics Experiments (Springer-Verlag, New York, LLC, 1994).

[55] S. Agostinelli et al., Nucl. Instrum. Methods Phys. Res., Sect. A 506, 250 (2003).

[56] J. Zhang, Ph.D. thesis, Old Dominion University, 2010, http://www.jlab.org/Hall-B/general/thesis/JixieZhang_thesis. pdf

[57] N. Guler, Ph.D. thesis, Old Dominion University, 2009, http://www.jlab.org/Hall-B/general/thesis/Guler_thesis.pdf

[58] M. Osipenko, A. Vlassov, and M. Taiuti, CLAS-NOTE 2004020, 2004, https://misportal.jlab.org/ul/Physics/Hall-B/clas/ viewFile.cfm/2004-020.pdf?documentId $=84$

[59] D. Abbott et al., Eur. Phys. J. A 7, 421 (2000).

[60] L. W. Mo and Y. S. Tsai, Rev. Mod. Phys. 41, 205 (1969).

[61] M. Lacombe et al., Phys. Lett. B 101, 139 (1981).

[62] S. J. Brodsky, H.-C. Pauli, and S. S. Pinsky, Phys. Rep. 301, 299 (1998).

[63] G. Kubon, H. Anklin, P. Bartsch, D. Baumann, W. U. Boeglin et al., Phys. Lett. B 524, 26 (2002).

[64] S. Galster, H. Klein, J. Moritz, K. H. Schmidt, D. Wegener et al., Nucl. Phys. B 32, 221 (1971).

[65] P. E. Bosted and M. E. Christy, Phys. Rev. C 77, 065206 (2008).

[66] K. Abe et al., Phys. Rev. D 58, 112003 (1998).

[67] R. Brun, M. Hansroul, and J. C. Lassalle (1982), GEANT Users Guide (CERN DD/EE/82).

[68] N. Baillie, Ph.D. thesis, College of William and Mary, 2010, http://www.jlab.org/Hall-B/general/thesis/Baillie_thesis.pdf

[69] M. E. Christy, N. Kalantarians, J. J. Ethier, and W. Melnitchouk (unpublished).

[70] M. E. Christy and P. E. Bosted, Phys. Rev. C 81, 055213 (2010).

[71] See Supplemental Material at http://link.aps.org/supplemental/ 10.1103/PhysRevC.89.045206 for tables of numerical results.

[72] S. Bueltmann et al., Jefferson Lab Experiment E12-10-102, 2010.

[73] Jefferson Lab Experiment CLAS Database, http://clasweb.jlab. org/physicsdb 